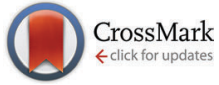

Cite this: Phys. Chem. Chem. Phys., 2014, 16, 21183

\title{
Fluorescence from graphene oxide and the influence of ionic, $\pi-\pi$ interactions and heterointerfaces: electron or energy transfer dynamics
}

\author{
Sesha Vempati*a and Tamer Uyar*ab
}

\begin{abstract}
2D crystals such as graphene and its oxide counterpart have sought good research attention for their application as well as fundamental interest. Especially graphene oxide (GO) is quite interesting because of its versatility and diverse application potential. However the mechanism of fluorescence from GO is under severe discussion. To explain the emission in general two interpretations were suggested, viz localization of $\mathrm{sp}^{2}$ clusters and involvement of oxygeneous functional groups. Despite this disagreement, it should be acknowledged that the heterogeneous atomic structure, synthesis dependent and uncontrollable implantation of oxygen functional groups on the basal plane make such explanations more difficult. Nevertheless, a suitable explanation enhances the applicability of the material which also enables the design of novel materials. At this juncture we believe that given the complexity in understanding the emission mechanism it would be very useful to review the literature. In this perspective we juxtapose various results related to fluorescence and influencing factors so that a conclusive interpretation may be unveiled. Apparently, the existing interpretations have largely ignored the factors such as self-rolling, byproduct formation etc. Vis-a-vis previous reviews did not discuss the interfacial charge transfer across heterostructures and the implication on the optical properties of GO or reduced graphene oxide ( $\mathrm{rGO}$ ). Such analysis would be very insightful to determine the energetic location of sub band gap states. Moreover, ionic and $\pi-\pi$ type interactions are also considered for their influence on emission properties. Apart from these, quantum dots, covalent modifications and nonlinear optical properties of GO and rGO were discussed for completeness. Finally we made concluding remarks with outlook.
\end{abstract}

Received 25th July 2014, Accepted 29th August 2014

DOI: $10.1039 / c 4 c p 03317 e$

www.rsc.org/pccp such as ZnO. ${ }^{16-22}$ A control on the reduction level enables the band gap tunability ${ }^{23}$ while its solution processability to make large area thin films is worth mentioning. ${ }^{24}$ The band gap tunability permits its application in mid-IR range photodetectors. Furthermore GO is integrated with silicon ${ }^{8}$ which suggests its suitability in industry. On the other hand biomedical applications include cell imaging, ${ }^{25}$ drug delivery, ${ }^{25,26}$ photothermal therapy and photoacoustic imaging, ${ }^{27}$ detection of $\mathrm{Cu}^{2+}$ ions, ${ }^{28}$ alcohol sensors, ${ }^{29}$ biosensors, ${ }^{3,30}$ in vivo toxicology effects ${ }^{31}$ etc. See a review article by Morales-Narvaez et al. for optical bio sensing applications of GO. ${ }^{30}$ Environmental remedies include photocatalysts $^{29,32,33}$ when combined with semiconductors such as $\mathrm{ZnO}, \mathrm{ZnS},{ }^{29}$ titanosilicate $^{33}$ etc. It is notable that the presence of another semiconductor is vital; hence the role of GO or reduced graphene oxide $(\mathrm{rGO})$ is to delay the recombination process in the semiconductor. ${ }^{32}$ Fig. 1 (top) shows the number of publications against year. We can see the intensity of research in the recent past on GO and related materials. In Fig. 1 (bottom) we have created a tabular form in which the distribution of research areas against the number of publications is given. These data are 


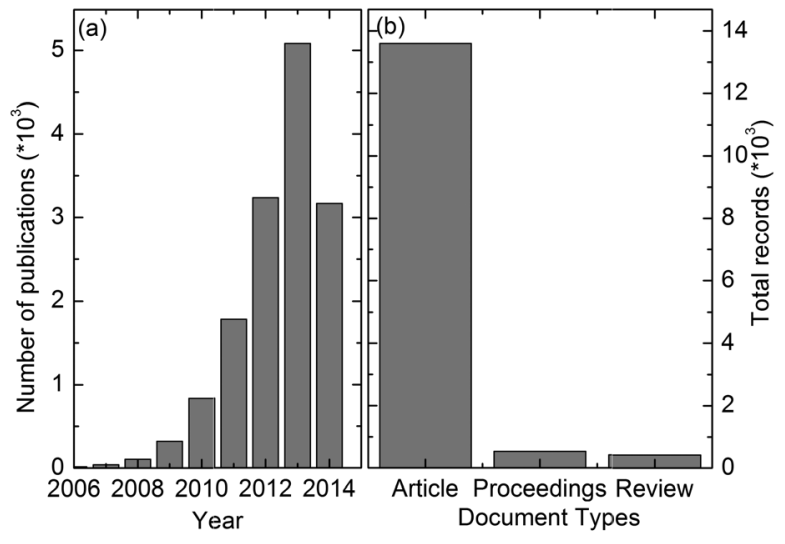

\begin{tabular}{|l|c|}
\hline Research Areas & Records \\
\hline Chemistry & 8854 \\
\hline Materials science & 7176 \\
\hline Science technology other topics & 3963 \\
\hline Physics & 3864 \\
\hline Electrochemistry & 1528 \\
\hline Engineering & 1058 \\
\hline Energy fuels & 968 \\
\hline Polymer science & 646 \\
\hline Biophysics & 295 \\
\hline $\begin{array}{l}\text { Biotechnology applied } \\
\text { microbiology }\end{array}$ & 248 \\
\hline Environmental sciences ecology & 215 \\
\hline Instruments instrumentation & 215 \\
\hline Optics & 207 \\
\hline Biochemistry molecular biology & 151 \\
\hline $\begin{array}{l}\text { Metallurgy metallurgical } \\
\text { engineering }\end{array}$ & 113 \\
\hline
\end{tabular}

Fig. 1 (top) (a) Number of publications against year and (b) shows the number of articles, reviews and proceedings that have appeared until now. Table (bottom) shows the distribution of research areas against number of publications. Data analyzed from web of knowledge as of 7th July 2014, key word for (a) graphene oxide.

convincing that the research interest in GO is constantly growing given its applicability in a range of research areas.

Fluorescence from graphene should be phonon assisted ${ }^{34}$ because of its zero band gap. In clear contrast, GO and rGO has shown NIR, visible and UV fluorescence $e^{15,25,26,35-39}$ with a quantum efficiency of $6.9 \% .{ }^{40}$ Luminescence of GO is also reported in red and NIR regions ${ }^{26,38}$ which can result from the presence of multilayered and aggregated flakes. ${ }^{36}$ Importantly, the mechanism which describes the fluorescence of GO or rGO is under severe discussion suggesting two different interpretations. One of them is the localization of $\mathrm{sp}^{2}$ clusters where the quantum confinement effect splits the energy bands and the recombination of $\mathrm{e}-\mathrm{h}$ pairs gives luminescence. The second explanation involves O2p orbital where the $\mathrm{CB}$ of the localized $\mathrm{sp}^{2}$ domains can be assigned to the $\pi^{*}$ orbitals, while the VB changes from the $\pi$ to the $\mathrm{O} 2 \mathrm{p}$ orbitals. In the former case, oxygen-related functional groups are eliminated from the emission mechanism due to the enhancement of fluorescence upon reduction. ${ }^{15,36,39} \mathrm{O} 2 \mathrm{p}$ orbitals are eliminated despite the fact that the method of reduction plays a crucial role in case if the process enhances radiative or non-radiative paths. ${ }^{41}$ Interestingly, as mentioned earlier, the band gap of GO is controllable $^{23}$ via manipulation of the reduction level. However, rGO is associated with a set of defects such as remnant oxygen atoms, ${ }^{42}$ pentagon-heptagon pairs (Stone-Wales defects) ${ }^{43,44}$ and holes ${ }^{44,45}$ due to the loss of carbon from the basal plane. ${ }^{46}$ Especially with the chemical reduction, hydrazine is found to be efficient in removing in plane functional groups (epoxy and hydroxyl), however, the edge moieties (carboxyl and carbonyl) stay undisturbed. ${ }^{4-49}$ In addition, it is also found that hydrazine reduction creates new functional groups such as $\mathrm{C}=\mathrm{N}$ on the $\mathrm{rGO}^{50-53}$ To emphasize, it is vital to elucidate a suitable mechanism for the luminescence of GO and rGO. This should be able to explain the influence of various factors such as the reduction level against luminescence properties. An appropriate mechanism allows us to design new material combinations where GO and rGO can be further exploited.

In the present perspective we have avoided the GO-synthesis details, however, please refer to an earlier article in which various chemical methods are discussed in the view point of large-area thin-film electronics and optoelectronics. ${ }^{5}$ Structural, electronic, optical and vibrational properties of nanoscale carbons and nanowires are discussed in a review by Cole et al. ${ }^{4}$ Graphenebased nanomaterials in optical and optoelectronic applications were reviewed by Chang et al. ${ }^{54}$ Given the background and disagreements in interpreting the emission mechanism necessitates its understanding of the current state of art. Hence in this perspective we critically discuss various results from the literature in an attempt to provide a clear insight into those explanations. We also cover the role of prominent functional groups and the tunable band gap, the excitation dependent emission process, quantum dots (QDs), the doping-effect on the emission properties, nonlinear optical properties and the influence of noncovalent/covalent functionalization. Given the contextual nature, we have briefly discussed various reduction processes and their effects as well. We will see that during the reduction process removal of oxygen is as inevitable as the formation of other complex bonds. Furthermore we have discussed ionic interactions such as $\mathrm{pH}$ dependency and interaction with other ionic species including the $\pi-\pi$ type. Finally, heterointerfaces and the consequent charge transfer mechanism are discussed in relation to photovoltaics and nanocomposites.

\section{Reduction of graphene oxide}

In the context of applications a scalable method is demanding to produce potential materials such as GO or rGO. The excellent properties depicted by these materials require mass production within the lines of well established and industrially applicable procedures. Gra in its oxidized form is less conducting (of course depending on the level of oxidation) because of the distorted conjugation. It is important that we meet the above mentioned criteria of scalability and conductivity. In this context one of the 
ways forward is the reduction of exfoliated GO. We can retrieve the electrical properties of GO to an extent by chemical and thermal reductions. ${ }^{35}$ To date, the rGO sheets reduced by hydriodic acid and acetic acid have shown the best electronic conductivity (up to $30000 \mathrm{~S} \mathrm{~m}^{-1}$ ). ${ }^{55}$ The 'retrieval of conductivity' is not the main objective of this section. Nevertheless the methods discussed here are in fact correlated with the emission properties. For example, in a molecular dynamics simulation the formation of highly stable carbonyl and ether groups is inevitable in a thermal reduction process. ${ }^{56}$ Hence the optical properties depicted by thermally reduced GO should consider the presence/ formation of these functional groups and the associated influence on the emission properties. In the following we have mentioned some of the techniques such as thermal, ${ }^{35,57}$ photo-thermal ${ }^{58}$ and chemical $^{6,35,59}$ reductions. Various other reducing agents and techniques can be seen from ref. 5. For the following reagents, see the cross references in the given citation. Ammonia, $\mathrm{NaBH}_{4}$, supercritical water, sugar and ascorbic acid; ${ }^{60}$ bovine serum albumin, bacterial respiration and hydriodic acid; ${ }^{61}$ hydroquinone, strong alkaline media, sulfur-containing compounds and amines; ${ }^{62}$ electrochemical and photographic camera flash. ${ }^{63}$ Reduction in principle decreases the density of oxygeneous functional groups, while the selectivity is of course process dependent. The presence of residual oxygen-containing functional groups and defects are detrimental for various applications. These active sites make the surface reactive and provide the tunability in electronic and optoelectronic properties via chemical reactions, ${ }^{36,43,64}$ including their incorporation in nanocomposites. ${ }^{65}$

When compared to hydrazine, hydriodic acid is less toxic and may be employed for the mass production of rGO. Controlled ozone treatment can tune the electrical and optical properties of graphene ${ }^{66}$ via oxidation. Thermal reduction is another versatile and industrially applicable process to reduce GO. ${ }^{35}$ Low temperature thermal reduction is implemented on large area self assembled GO films. ${ }^{57}$ Furthermore, in photothermal reduction UV light impinges on the samples which are simultaneously subjected to heating. This is a quite interesting method where a precise control of the reduction level can be obtained, ${ }^{58}$ especially in the lab scale environment for synthesizing novel derivatives of GO.

In the context of chemical reduction, hydrazine and its derivatives are rather potential reducing agents which were extensively studied in the literature. ${ }^{35}$ The important consequences of employing hydrazine in vapor or liquid phase are discussed in Section 3.2. To draw readers' attention to one of the key features, a study by Mathkar et $a{ }^{59}$ shows the band gap tunability by simply varying the exposure time of hydrazine vapor (will be discussed, Fig. 5). Oxygen plasma treatment is a better method in some aspects when compared to that of hydrazine. The oxygen plasma treatment creates much cleaner $\mathrm{rGO}^{67}$ while converting the epoxy groups into carbonyl groups though limited to the surface for a multilayered sample. Interestingly, oxygen plasma treatment can convert non-emitting graphene into a broad red-NIR emitting layer $^{68}$ with spatial uniformity. While hydrazine treatment is prone to create $\mathrm{C}=\mathrm{N}$ bonds. ${ }^{50,53}$

\section{Emission properties of GO}

\subsection{Fluorescence of luminescence?}

Several authors refer the emission from GO as photoluminescence (PL). However, given the time scales of the decay process it would be appropriate to refer the emission as fluorescence (PL occurs in the order of ps). For example, lifetimes are below $6 \mathrm{~ns}$ for multicolour fluorescent GO which is synthesized by cleaving CNT upon oxidation. ${ }^{69}$ Some examples of decay times for various combinations of GO or rGO with other materials are tabulated in Table 1. Also the details of excitation and emission wavelengths were given where available. From the table, it is clear that the decay times are in the order of nanoseconds. Nevertheless it is notable that the total decay curve might an integral of more than one decay process. ${ }^{70}$ It is important to note that the number of components is determined by the chemistry of the material and the relative stability of the intermediate states. A better understanding of the emission

Table 1 Decay times for various combinations of GO or rGO and the mechanism if attributed

\begin{tabular}{|c|c|c|c|c|c|c|c|c|}
\hline \multirow[b]{2}{*}{ S. no } & \multirow[b]{2}{*}{ Compound } & \multirow{2}{*}{$\begin{array}{l}\text { Excitation } \\
\lambda_{\mathrm{ex}}(\mathrm{nm})\end{array}$} & \multirow{2}{*}{$\begin{array}{l}\text { Emission } \\
\lambda_{\mathrm{em}}(\mathrm{nm})\end{array}$} & \multicolumn{3}{|l|}{ Decay time (ns) } & \multirow[b]{2}{*}{ Mechanism/comment } & \multirow[b]{2}{*}{ Ref. } \\
\hline & & & & $\tau_{1}$ & $\tau_{2}$ & $\tau_{3}$ & & \\
\hline 1 & GO from cleaved CNT & 365 & $38-690$ & 5.1 & & & Localized $\mathrm{sp}^{2}$ clusters & 69 \\
\hline \multirow[t]{4}{*}{2} & rGO & 318 & All & 5 & 1.2 & 0.2 & $\pi-\pi$ type noncovalent attachment & 70 \\
\hline & $\mathrm{Rb}$ & 375 & $400-700$ & 4.76 & & & & \\
\hline & rGO-Rb noncovalent & 358 & 440 & & & & & \\
\hline & 362 & 460 & & & & & & \\
\hline \multirow[t]{2}{*}{3} & GO QDs & NA & NA & $\sim 5.4$ & & & & 28 \\
\hline & GO QDs/Cu ${ }^{2+}$ & NA & NA & $\sim 5.4$ & & & Complexation (static quenching) & \\
\hline \multirow[t]{4}{*}{4} & $\mathrm{P}^{+}$ & $438^{a}$ & 640 & $\sim 20$ & & & & 91 \\
\hline & $\mathrm{GO} / \mathrm{P}^{+}$ & $438^{a}$ & 640 & 1.2 & 6 & & $\begin{array}{l}\mathrm{e}^{-} \text {and/or energy transfer from } \mathrm{P}^{+} \text {to } \mathrm{GO} \text {, donor } \\
\text { acceptor complex }\end{array}$ & \\
\hline & $\mathrm{P}^{-}$ & $438^{a}$ & 640 & $12<\tau_{1}<20^{b}$ & & & & \\
\hline & $\mathrm{GO} / \mathrm{P}^{-}$ & $438^{a}$ & 640 & & & & No interaction: repulsion between similar charges & \\
\hline \multirow[t]{3}{*}{5} & P3HT & $400^{c}$ & 575 & 0.748 & & & Covalent bonding: $\pi-\pi$ interaction dynamic quenching & 92 \\
\hline & $\mathrm{GO} / \mathrm{P} 3 \mathrm{HT}$ & $400^{c}$ & 575 & 0.532 & & & and forming a non-fluorescent ground-state complex & \\
\hline & $\mathrm{rGO} / \mathrm{P} 3 \mathrm{HT}$ & $400^{c}$ & 575 & 0.351 & & & Charge pairs are injected into GO as fast as $1.4 \mathrm{ps}$ & \\
\hline
\end{tabular}


properties can perhaps suggest an appropriate number of decay constants.

\subsection{Mechanism of fluorescence}

If fluorescence has to occur in Gr then it must be assisted by phonons ${ }^{34}$ because of its zero band gap. While in the case of GO and rGO with heterogeneous atomic and electronic structures depicted UV, visible and NIR fluorescence. ${ }^{6,15,25,26,35-39}$ On the other hand, UV-Vis emission from carbon based materials (amorphous, ${ }^{71-73}$ disordered carbons ${ }^{74-76}$ ) is known. However, band gap tunability and solution processability of GO enables its versatility in various applications. ${ }^{6}$ Previously (Section 3.1, Table 1) we have broadly seen the emission wavelengths and their decay times of GO and rGO in pure form or when attached to other functional materials via covalent or noncovalent means. Note that as-synthesized GO did not emit light at all instances. ${ }^{37,77}$ On the other hand emission at specific wavelengths, for example, $440 \mathrm{~nm},{ }^{15} 505 \mathrm{~nm}^{31}$ and the blue-UV region, ${ }^{15,36,39}$ was observed. The emission wavelengths of GO depend on the functional groups, ${ }^{45} \mathrm{pH}^{69,78-82}$ and its combination with other materials such as PANI-nanorods, ${ }^{83} \mathrm{MB},{ }^{84}$ tetra-amino porphyrin, ${ }^{85} \mathrm{PEG}^{25,26}$ etc. Since the emission from GO is dependent on various factors, one should go deeper to understand the mechanism. Strong heterogeneity in the atomic and electronic structure makes the emission process quite complex. Fluorescence from GO arises from the recombination of $\mathrm{e}-\mathrm{h}$ pairs in localized electronic states of various configurations. Having said that, the exact mechanism is still unknown. However, researchers have attempted to provide some crucial insights and interpretation for their observations, which we summarize below. Before we go into those details, excitation dependent fluorescence will be discussed.

GO depicts excitation dependent emission as observed by many groups. ${ }^{86-88}$ The reason for excitation dependency is that different transitions are possible from the CBM and nearby localized states to the wide-range VB. While the lack of emission for the excitation above the band gap ${ }^{5,37}$ can be due to the fact that the excitation energy is either dissipated as heat or injected into the adjacent metallic phase of the carbon sheet. ${ }^{77}$

The emission from GO is in clear contrast to the general semiconductors. In the case of general semiconductors the band edge transition and subsequent recombination yields PL. One of the explanations given for the fluorescence of GO is as follows. The fluorescence from GO arises from e/h recombination in localized $\mathrm{sp}^{2}$ electronic states which are confined within the $\mathrm{sp}^{3}$ matrix, i.e. confinement of $\pi$-electrons (please see Section 3.4 for size dependent effects). ${ }^{71-73}$ Although $\mathrm{sp}^{2}$ clusters are under quantum confinement, ${ }^{6}$ there are no discrete energy levels, however the local energy gap is determined by the cluster size. It means that for a given sample, the size differences in the clusters produce multiple wavelengths. Hence the attribution of wavelengths to the 'average cluster size' needs to be handled carefully especially when wavelength specific applications are considered. It is notable that GO gives fluorescence when the concentration of $\mathrm{sp}^{2}$ cluster is optimum, ${ }^{36}$ passivated reactive sites, ${ }^{89}$ chemical bonding with fluorescent ions, ${ }^{90}$ or in the form of QDs. ${ }^{37}$ The typical electronic structure of GO can be schematized as

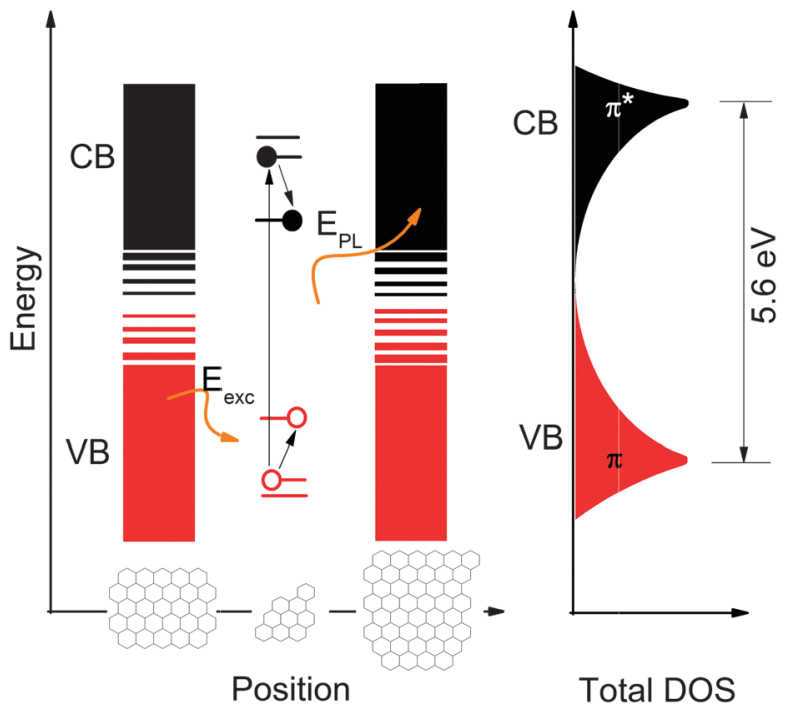

Fig. 2 Schematic band structure of GO. Smaller $\mathrm{sp}^{2}$ domains have a larger energy gap due to a stronger confinement effect. DOS-electronic density of states. Figure redrawn after ref. 36.

shown in Fig. 2, where the black arrows denote the transitions of electrons and holes under suitable illumination $\left(E_{\text {exc }}\right)$. Upon absorbing $E_{\text {exc }}$, e-h pairs are created followed by non-radiative relaxation and radiative recombination emitting $E_{\mathrm{PL}}$. The emission bands are dependent on electronic band gaps of $\mathrm{sp}^{2}$ clusters (mixture of $\mathrm{sp}^{2}$ and $\mathrm{sp}^{2}$ bonding). ${ }^{71,93,94}$ Moreover the band gap is associated with the size, shape, and fraction of the $\mathrm{sp}^{2}$ clusters located within the $\mathrm{sp}^{3}$ matrix. ${ }^{36}$ For instance smaller $\mathrm{sp}^{2}$ clusters depict wider energy gaps because of the relatively higher quantum confinement effect. From the given range of $\mathrm{sp}^{2}$ cluster size, it is hard to distinguish the features depicted by each cluster. Hence an integral effect is generally seen. Most of the synthesis methods are not very successful in producing GO with a controlled or predetermined cluster size. Further details on how to calculate the cluster size are given in Section 3.4.

There is an alternative explanation given in the literature for fluorescence from GO. ${ }^{37}$ In this investigation the authors have used a hydrothermal technique to cut GO sheets into QDs which emit blue color. The authors suggested that the emission occurs from zigzag sites, where their ground state is in a triplet state similar to carbene. This can be described as $\sigma^{1} \pi^{1}$ as shown in Fig. 3. The argument is based on the fact that the fluorescence originates from the oxygeneous functional groups as seen earlier in the case of carbon nanoparticles, ${ }^{75,76,95}$ functionalized $\mathrm{CNTs}^{74,96}$ and surface-oxidized Si nanocrystals. ${ }^{97}$ However, Loh et $a{ }^{6}{ }^{6}$ suggest that the enhancement of fluorescence with reduction excludes oxygen containing functional groups from the possible origin. ${ }^{15,36,39}$ Although it is convincing that the exclusion is drawn based on the references ${ }^{15,36}$ and, ${ }^{39}$ according to Loh et $a .^{6}$ the localized $\mathrm{sp}^{2}$ cluster and structural defects during the reduction ${ }^{98}$ seemed to be a more suitable explanation for the origin and the enhancement of blue fluorescence. ${ }^{36}$ On the other hand Chien et al. suggested that the visible emission might arise from defect related states within an interface. ${ }^{58}$ 


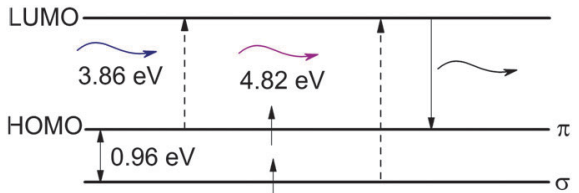

Fig. 3 Schematic of the electronic structure at the zigzag edge site similar to carbene. Dashed (excitation) and solid arrows (relaxation) for $\sigma$ - and $\pi$ states. Figure redrawn after ref. 37

Based on the following reasons it is vital to discuss and reconsider the previous argument ( $\mathrm{sp}^{2}$ cluster localization) to explain the emission from GO. Upon reduction, it is true that the density of oxygen containing functional group decreases. The fluorescence intensity or QY, of course, depends on various factors such as absorption efficiency and the balance between radiative and non-radiative recombinations. Oxygen containing functional groups are eliminated from the emission mechanism due to the enhancement of fluorescence upon reduction. ${ }^{15,36,39}$ Conjointly, the method of reduction is a key factor to consider, in case if the process enhances radiative or non-radiative paths. For example, in ref. 15 three different methods were employed to reduce the GO yielding Gra. Viz. thermal exfoliation at high temperatures, heating nanodiamond in an inert atmosphere and arc discharge of graphite electrodes in the presence of $\mathrm{H}_{2} / \mathrm{He}$. This few-layer-Gra was subjected to acid treatment under microwave irradiation to yield GO. Subrahmanyam et al. ${ }^{15}$ suggest blue emission centred around $400 \mathrm{~nm}$ from as-prepared Gra samples, which implies that complete conversion of GO into Gra did not take place through the above three reduction processes. To emphasize, fluorescence in Gra is assisted by phonons. ${ }^{34}$ Apart from the above mentioned differences, the intensity scale on the fluorescence spectra or the details of QY were not given by the authors in ref. 15. In ref. 36 the authors have used hydrazine for the reduction of GO. It is undisputed that hydrazine treatment decreases the density of oxygen containing functional groups. However some of the reports suggest enhancement of blue fluorescence and quenching of the initial yellow-red fluorescence $^{39}$ in addition to the following points. In the case of exposure to hydrazine vapor the functional groups are reduced in the following order as suggested by Mathkar et al. ${ }^{59}$ (i) phenol and carbonyl groups are the first to be reduced, then (ii) epoxide moieties and finally (iii) tertiary alcohols. In this context it is notable that the electron withdrawing capacity (acidity) depends on the functional group, thereby a variation in the electron DOS of rGO is expected. Furthermore, hydrazine treatment can form $\mathrm{C}=\mathrm{N}^{50,99-101}$ bonds on rGO. It is also found that the fluorescence intensity of GO is greatly enhanced with no spectral shift after a short exposure of hydrazine vapors. ${ }^{36}$ During hydrazine monohydrate reduction XPS has evidenced $\mathrm{C}=\mathrm{N}$ functional groups, ${ }^{99}$ resulting from a reaction as explained in the ref. 100 and 101. Furthermore, the reduction of GO is accompanied by some nitrogen incorporation from the reducing agent $(\mathrm{C} / \mathrm{N}=$ 16.1 by elemental analysis). This is presumably through a reaction of hydrazine hydrate with the carbonyl groups of GO. ${ }^{51}$ Notably, the incorporation of ' $\mathrm{N}$ ' in the rGO is suggested to take place via other functional groups such as lactones, anhydrides, quinones with which hydrazine can react. ${ }^{51}$ Hydrazine is found to be efficient to remove in plane functional groups such as epoxy and hydroxyls, however, the edge moieties such as carboxyl and carbonyl stay intact. ${ }^{47-49}$ Another study suggests that the hydroxyls on the basal planes of GO were not removed by hydrazine hydrate even at elevated temperature. ${ }^{50}$ Furthermore this study also suggests that the carbonyl and carboxylate groups formed the $\mathrm{C}=\mathrm{N}$ bonds of hydrazones. ${ }^{50}$ After hydrazine vapour treatment, ${ }^{53}$ incorporation of nitrogen at a substantial level was confirmed by XPS analysis and attributed to partial reduction of carbonyl groups to hydrazone groups. ${ }^{51,52}$ It is also important to consider the synthesis method of GO against the hydrazine reduction process as the former plays a major role in determining the functional groups, density and their physical location on the graphitic plane. As the reduction takes place the distance between the sheets decreases because of the $\pi-\pi$ interactions. Given the discrepancy in the literature, it is highly recommended that the effect of hydrazine on the type (synthesis method) of GO requires thorough investigation.

In the context of GO QDs, the fluorescence intensity from as synthesized QDs is higher than its annealed $\left(200{ }^{\circ} \mathrm{C}\right.$ in vacuum) counterpart apart from a blue shift. ${ }^{36}$ During the thermal annealing process, formation of intermediate phases was observed by Jeong et al. ${ }^{102}$ These phases were attributed to the conversion of hydroxyl groups into epoxide and carboxyl groups. As a consequence the interlayer distance is increased and the carbon backbone switches to a $\mathrm{sp}^{3}$ structure. ${ }^{102}$ Similar observation and attribution is suggested in a study by Cuong et al. ${ }^{103}$ Furthermore in molecular dynamics simulations the formation of highly stable carbonyl and ether groups was observed in the thermal reduction process. ${ }^{56}$ Hence the optical properties depicted by the thermally reduced GO should consider the presence of these functional groups and the associated influence on the emission properties.

The existence of the $\mathrm{O} 2 \mathrm{p}$ level and its active participation were discussed in the context of the $\mathrm{TiO}_{2} / \mathrm{GO}$ heterointerface (Fig. 18d). ${ }^{77}$ In this study IOT (reduced symmetry at the interface, ${ }^{104}$ type-II fluorescence ${ }^{105}$ ) was observed between $\mathrm{TiO}_{2}$ and the $\mathrm{O} 2 \mathrm{p}$ of GO. Under suitable illumination, the electrons localize in the $\mathrm{CB}$ of $\mathrm{TiO}_{2}$ while the holes can either relax to a defect level or injected to the $\mathrm{O} 2 \mathrm{p}$ level. The optical recombination of electrons from $\mathrm{CB}$ of $\mathrm{TiO}_{2}$ with that of holes in $\mathrm{O} 2 \mathrm{p}$ levels of GO gives fluorescence (IOT). The details of IOT will be discussed more elaborately in Section 7.2.

We point out another important study by Zhang et al. ${ }^{106}$ in which the authors have studied the optical properties against the self-rolling effect of chemically derived graphene sheets. For concentrations less than $10 \mathrm{mg} \mathrm{mL}^{-1}$, these sheets have shown self-rolling, and aggregated at higher than the said value. The earlier studies in which the fluorescence quenching effect is reported may be reconsidered, as the rolling of sheets severely influences the electronic absorption and emission properties. As a matter of fact, numerous studies evidenced that Gra acts as an electron reservoir, where the photogenerated electrons are collected from an adjacent/accompanying semiconductor. ${ }^{29,32,33}$ 
Aggregated sheets have shown a clear deviation from the BeerLambert law. Apart from these, the absorptivity was decreased and spectral shapes were changed. Rolled sheets depicted new absorption (at 500 and $960 \mathrm{~nm}$ ) and emission (after $500 \mathrm{~nm}$ ) bands with decreased emission efficiency. ${ }^{106}$ Furthermore this study also suggests that the emission mechanism for single and double layered GO or rGO needs to be re-examined. Self-rolling can be avoided by choosing an appropriate solvent, however, it associates another complexity such as 'dielectric constant' as it plays a key role in the emission process and its energy. ${ }^{6}$ Having said that, for sheet dimensions in the range of 1-10 $\mu \mathrm{m}$, their dispersion and solid sample have shown comparable fluorescence. ${ }^{38}$ Extending the argument of self-rolling, with the increasing reduction level the $\pi-\pi$ interaction among the sheets also increases and hence the carrier dynamics may be influenced significantly. Finally, similar to the effects from 'hydrazine reduction', the effect of 'dielectric constant' should be investigated further.

In the band diagram of second explanation for the fluorescence, the $\mathrm{CB}$ of the localized $\mathrm{sp}^{2}$ domains are assigned to the $\pi^{*}$ orbitals, while the VB changes from the $\pi$ to O2p orbitals. ${ }^{41}$ Ref. 41 contains discussion of the results from local DFT simulations via first-principles. The energy of the indirect band gap increases with the increasing degree of oxidation, e.g. $\sim 2.7-3.2 \mathrm{eV}$ for the GO samples studied in ref. 107. Relatively higher band gap causes extremely weak absorption for GO in the visible range. ${ }^{90,107}$ The changing of $\mathrm{VB}$ from the $\pi$ to the $\mathrm{O} 2 \mathrm{p}$ orbitals is also suggested by Jeong et al. where the HOMO level shifts downwards opening the band gap. ${ }^{108}$ It will be very useful, if wavelength selective photodetectors based on GO or rGO are studied while combining the well understood materials. This allows us to elucidate the energetic location of bands and carrier dynamics there in. ${ }^{18}$

\subsection{Role of prominent functional groups, tunable band gap}

In the previous section we mainly discussed two mechanisms that may describe the fluorescence in GO and rGO. In this section we will see how the functional groups inflect the optical properties. ${ }^{45}$ It is vital because when GO is reduced with hydrazine (Section 3.2) the oxygen related functional groups follow a sequence ${ }^{59}$ where phenol and carbonyl groups are the first and tertiary alcohols are the last to be reduced. An experimental investigation of GO and rGO has also suggested that the oxygeneous functional groups play a major role in determining the band gap. ${ }^{23} \mathrm{~A}$ mixture of oxygen and hydroxyl groups with a coverage of $100 \%, 75 \%, 50 \%$ depicted band gaps of $\sim 2.8,2.1$ and $1.8 \mathrm{eV}$, respectively. ${ }^{23}$ The control of the density and coverage of these functional groups allows us to tune the band gap of rGO. In a study by Johari et al. ${ }^{45}$ ab initio DFT based simulations were performed to understand the electronic and optical properties of periodic structures. In this investigation ${ }^{45}$ GO with different coverage densities and compositions of functional groups (epoxides, hydroxyls and carbonyls) were studied. The key findings were as follows. (i) Optical band gap decreases rapidly (4.0 to $0.3 \mathrm{eV}$ ) with an increase in the size of the hole or defect in the case of carbonyl groups (an $\mathrm{O}$ to
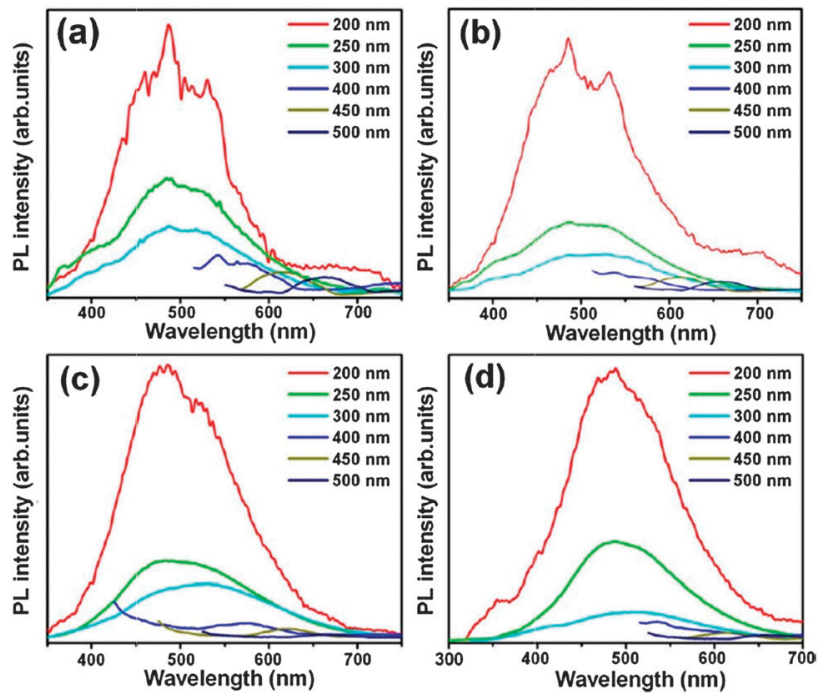

Fig. 4 Fluorescence spectra for (a) GO-1, (b) GO-2, (c) GO-3, and (d) GO4 films at different $\lambda_{\text {ex }}$. Reproduced with permission from ref. 67.

$\mathrm{C}$ ratio from 0 to $37.5 \%)$. When epoxy and hydroxyl functional groups vary from 25 to $75 \%, \pi+\sigma$ plasmon is found to depict a significant blue shift $(\sim 1.0-3.0 \mathrm{eV})$ unlike the $\pi$ plasmon peak which is less sensitive. Furthermore, the increase in carbonyl groups on the basal plane creates holes and consequently the $\pi$ plasmon peak is shifted by $\sim 1.0 \mathrm{eV}$ when compared to that of the pristine Gra. This study shows that the earlier argument of method of synthesis is an important factor to consider, where the density of these oxygeneous functional groups varies depending on the process. Taking the discussion a step forward, if the epoxy groups on GO are converted (oxygen plasma treatment) into carbonyl groups ${ }^{67}$ apart from the excitation dependency, the luminescence spectra depicted distinct features (Fig. 4). As the oxygen pressure increases (GO-2 to GO-4: $\mathrm{sp}^{3}$ hybridization increases) the shoulder at $530 \mathrm{~nm}$ disappears apart from a spectrally invariant emission at $487 \mathrm{~nm}$. Clusters of larger sizes are more prone to oxidation introducing nonradiative paths (epoxy \& carbonyl) and dangling bonds which result in quenching of emission at longer wavelengths $(550-650 \mathrm{~nm})$. Interestingly, the QY increases from GO-2 to GO-4 compared to GO- $1 .{ }^{109}$ The emission has occurred from a range of GO dimensions, where red to NIR is observed in nanosized aqueous GO dispersions. ${ }^{25,26}$ Note that the GO in these two cases is functionalized with PEG.

Experimentally a control on the reduction of functional groups of GO is achieved through hydrazine vapor exposure. It enables the band gap tunability from 3.5 to $1 \mathrm{eV}$ (Fig. 5). ${ }^{59}$ Refer to Section 3.2 for more details related to this method of reduction. Within the first $8 \mathrm{~h}$ of hydrazine exposure the optical band gap is seen to fall rapidly from a starting point of $3.5 \mathrm{eV}$. Precise control on the reduction time yields the band gap that we require, however, the density of functional groups cannot be controlled with this process. As an aside, spectroscopic ellipsometry can be employed to estimate the band gap by applying the Lorentz oscillator model which provides accurate energy level distribution in GO or rGO. ${ }^{23,110}$ Apart from UV-Vis spectroscopy, 


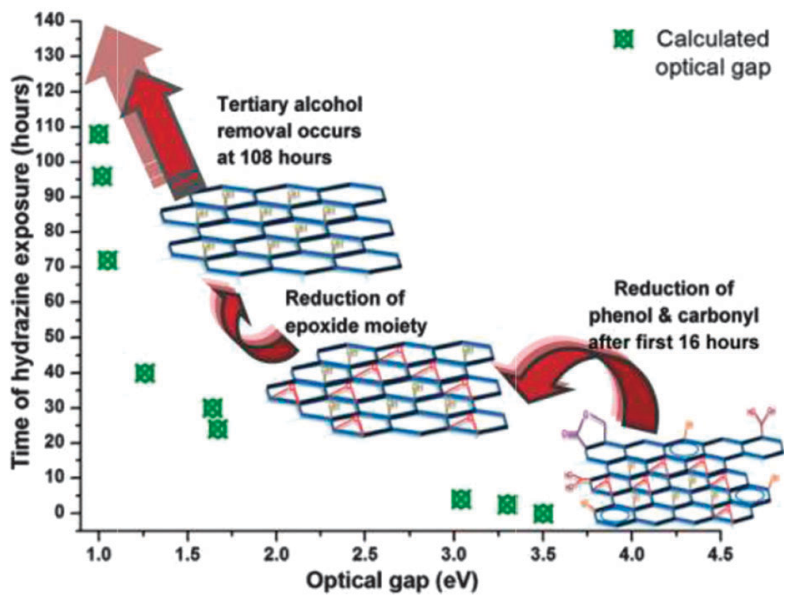

Fig. 5 Band gap modulation upon exposure to hydrazine vapors along with a schematic rGO structure at selected time intervals. Reproduced with permission from ref. 59 .

cyclic voltammetry can be used with which the edges of $\mathrm{CB}$ and VB can be determined..$^{59}$ Crucially, it should be unveiled whether these techniques yield comparable results for GO and rGO in the background of their complex band structure. Controllable oxidation of Gra is also a subject of investigation ${ }^{111}$ however, Wang et al. did not provide an estimation of the band gap with reference to different oxidation levels.

\subsection{Quantum dots}

The applications of GO QDs have spread into biomedical engineering because of their size dependent emission properties. They are cell imaging, drug delivery, ${ }^{25}$ selective detection of $\mathrm{Cu}^{2+}$ ions ${ }^{28}$ etc. Notably the size dependent emission of GO QDs is similar to that of carbon QDs. ${ }^{75}$ GO QDs were synthesized variously. ${ }^{37,112}$ For example 1-4 nm sized QDs (referred to as graphene quantum dots in ref. 112) were synthesized from carbon fibers which not only offer a cheap alternative route but also a control of the size enables tunable fluorescence. ${ }^{112}$ In vivo toxicology effects are also studied for carboxylated GO QDs ${ }^{31}$ (Fig. 6). In Fig. 6 schematic of synthesis, TEM, DLS and fluorescence properties (at $505 \mathrm{~nm}$ ) were shown for carboxylated GO QDs. KB cells were treated with these carboxylated GO QDs and the corresponding CLSM images is shown. Density gradient ultracentrifuge is employed to obtain monodisperse GO $\mathrm{QDs}^{113}$ where the UV-Vis and fluorescence spectra revealed that the properties of samples are highly dependent on their sheet size and degree of oxidation. Eda $e t$ al..$^{36}$ attributed the emission to quantum confinement of $\mathrm{sp}^{2}$ clusters which in turn connects to its band gap. ${ }^{71,93,94}$ Moreover the band gap depends on the size, shape and fraction of the $\mathrm{sp}^{2}$ clusters. ${ }^{36}$ Initially the cluster size $\left(L_{\mathrm{a}}, \AA\right)$ was estimated by Tuinstra et al. ${ }^{114}$ in 1970 by an empirical relation as $L_{\mathrm{a}}=43.5\left(\mathrm{I}_{\mathrm{D}} / \mathrm{I}_{\mathrm{G}}\right)^{-1}$ which was later verified by Knight et al. ${ }^{115}$ with additional data points. Note that the method shown in ref. 114 underestimates the crystallite size if there is a dominant effect of small crystallites, despite it can be validated with the crystallinity from XRD. However, the linear relation suggests that the Raman intensity is proportional to the 'boundary' in the sample. ${ }^{114}$ UV-Vis ( $\mathrm{sp}^{2}$ clusters size $<1 \mathrm{~nm}$ ) and red-IR emission $\left(\mathrm{sp}^{2}\right.$ cluster
A
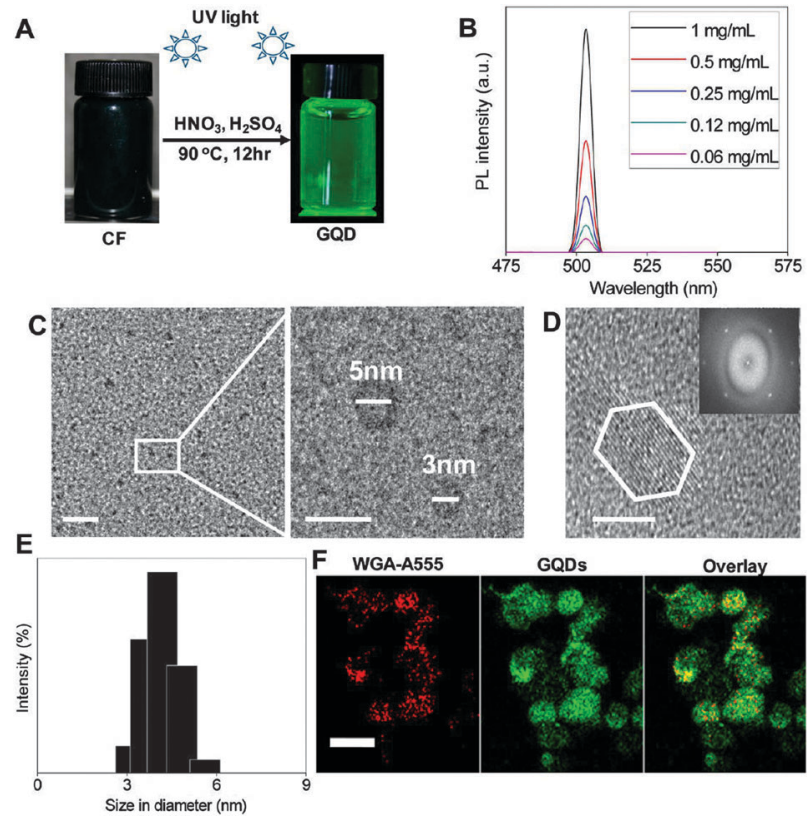

Fig. 6 (A) Synthesis and fluorescence of GO QDs, (B) fluorescence intensities at $505 \mathrm{~nm}$ wavelength, (C) TEM images; scale bar is $50 \mathrm{~nm}$ for the left image and $10 \mathrm{~nm}$ for the right image, (D) HR-TEM image (scale bar $=5 \mathrm{~nm}$ ) showing the edge structure of lattices formed in QDs, inset shows Fourier transformed image, (E) size distribution of the carboxylated Gr QDs measured by DLS and (F) CLSM images of KB cells treated with the carboxylated QDs (scale bar = $50 \mu \mathrm{m})$. Reproduced with permission from ref. 31 .

size $>2 \mathrm{~nm}$ ) are observed by Eda et al. ${ }^{36}$ As synthesized GO has a larger sp ${ }^{2}$ cluster size $(4.83 \mathrm{~nm})$ with a narrower band gap emitting in the green-to-red region. After annealing, the cluster size $(3.95 \mathrm{~nm})$ as well as emission intensity is decreased apart from a blue shift in the emission spectrum. Other studies have shown similar results for $\mathrm{sp}^{2}$ cluster sizes of $2.5-8 \mathrm{~nm} .^{5,38,43,71,94,103,116-121}$ The authors attributed the decreased cluster size to the nucleation of $\mathrm{sp}^{2}$ domains in the $\mathrm{sp}^{3}$ matrix. For the cases in which the thermal process is employed for the reduction the earlier discussed consequences should be considered (Section 3.2).

GO QDs (referred to as graphene quantum dots) were synthesized by Peng et al. ${ }^{112}$ where the variance in the size offers a tunable band gap and consequently the emission characteristics can be controlled. The UV-Vis absorption spectra were shown in Fig. 7 of GO QDs synthesized at 80, 100, and $120{ }^{\circ} \mathrm{C}$. See the inset of Fig. 7 for digital photographs under UV light. A clear blue shift is noticed from 330 to $270 \mathrm{~nm}$ with increasing synthesis temperature. The fluorescence spectra (Fig. 7b) can be understood from the average sizes, shape and defect densities. ${ }^{64}$ The size differences may cause variation in density and nature of $\mathrm{sp}^{2}$ sites, which results in varying band gap (3.90 to $2.89 \mathrm{eV}$ ).

Note that this trend is similar to the quantum confinement effect at lower particle sizes $(1-10 \mathrm{~nm}) .{ }^{122}$ From the PLE spectra two new transitions (at 284 and $318 \mathrm{~nm}$ ) were seen, where they can be considered as a transition from the $\sigma$ and $\pi$ orbital (HOMO) to the LUMO (Fig. 7c) in contrast to $\pi-\pi^{*}$ transition. In the case of carbine for a triplet ground state energy differences 


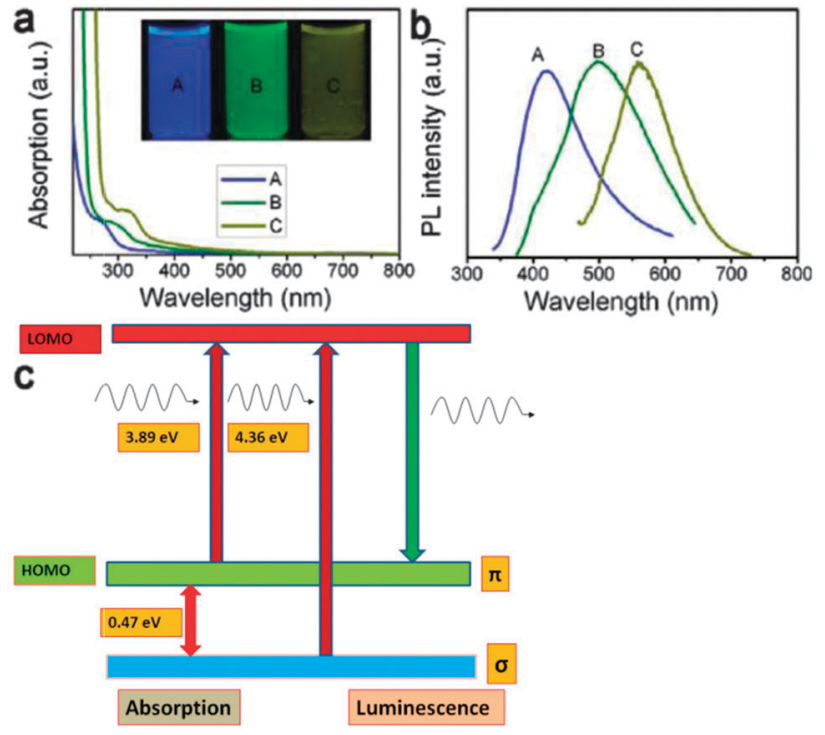

Fig. 7 (a) UV-Vis spectra of GO QDs (A-C), correspond to the reaction temperatures at 120,100 , and $80{ }^{\circ} \mathrm{C}$, respectively. Inset of panel a is a photograph of GO QDs under $365 \mathrm{~nm}$ illumination. (b) Fluorescence spectra for $\lambda_{\mathrm{ex}} 318$ (A), 331 (B), and $429 \mathrm{~nm}$ (C) and (c) electronic transitions of triple carbenes at zigzag sites observed in the optical spectra for blue emission. Reproduced with permission from ref. 112 while part $\mathrm{c}$ is taken from its supplementary information.

between the $\sigma$ and $\pi$ orbital should be below $1.5 \mathrm{eV},{ }^{123,124}$ where it is $0.47,0.82$, and $1.24 \mathrm{eV}$ for blue, green and yellow emission, respectively. Under alkaline conditions, the GO QDs emit strong fluorescence, while acidic conditions quench the PL, because the free zigzag sites are protonated while forming a complex. ${ }^{112}$ In general the quantum sized materials of course behave differently from their bulk counterparts. Nevertheless, $\mathrm{sp}^{2}$ clusters localized in the $\mathrm{sp}^{3}$ matrix of $1-10 \mu \mathrm{m}$ overall size is different from that of $\mathrm{sp}^{2}$ clusters in a quantum sized particle of 1-10 $\mathrm{nm}$. In the former case the localization is constrained within the $\mathrm{sp}^{3}$ matrix where the edge effects can be largely ignored. This is in clear contrast to the latter case where the edge effects are as prominent as the surrounding $\mathrm{sp}^{3}$ matrix.

QY can be calculated by comparing the integral intensity with constant absorbance. ${ }^{109}$ If we take a look at the QY of the GO, it is relatively low (6.9\% ref. 40$)$ at times as low as less than $1 \%{ }^{38}$ which can be because of two main factors. ${ }^{81}$ The presence of (i) isolated $\mathrm{sp}^{2}$ domains and (ii) reactive sites such as the epoxide groups inducing nonradiative recombination. It is expected that when the surface is modified, the reactive sites may be passivated and hence luminescence yield may improve. Defect states within the interfaces may cause nonradiative transition, which might reduce the emission intensity ${ }^{125}$ and thus the QY. In some cases no emission is observed until GO was subjected to specific modifications such as appropriate control of the $\mathrm{sp}^{2}$ cluster concentration, ${ }^{36}$ or surface passivation of the reactive sites. ${ }^{89}$

\subsection{Doping}

Similar to regular semiconductors ${ }^{16,21} \mathrm{GO}$ is subjected to doping. In this section we will discuss the effects of substitutional doping while that of surface electron transfer ${ }^{126,127}$ will be discussed later. Doping of GO is rather interesting and extensively investigated ${ }^{99,128,129}$ especially with nitrogen, ${ }^{99,129}$ boron, ${ }^{128}$ halogens ${ }^{130}$ etc. In the context of fluorine doping, a completely fluorinated graphene behaves as a thinnest insulator and the only stable stoichiometric graphene halide $\left(\mathrm{C}_{1} \mathrm{X}_{1}\right){ }^{130}$ Fluorinedoped rGO is reportedly a better substrate for surface enhanced Raman spectroscopy than unmodified rGO. Since F doped rGO or GO doesn't show any emission, we will not discuss their details. However, the reader is advised to refer to a recent review by Karlicky et al. ${ }^{130}$ In the process of doping, formation of other phases is an important issue. For example, $\mathrm{B}$ doping has resulted in the presence of $\mathrm{B}_{4} \mathrm{C}, \mathrm{B}_{\mathrm{C}}, \mathrm{BC}_{2} \mathrm{O}, \mathrm{BCO}_{2}$ and $\mathrm{B}_{2} \mathrm{O}_{3} \cdot{ }^{128}$ Recently, the energy-level structure of N-doped GO QDs was discussed. ${ }^{129}$ Simultaneous doping of B and N doping. is also possible, where GO is converted into boron carbonitride by substitutional doping. ${ }^{131}$ Interestingly, after the doping process $\left(\right.$ at $900{ }^{\circ} \mathrm{C}$ ), a significant amount of oxygen content in the GO is evidenced from XPS. Essentially the BN phase is formed within the GO matrix, $c f$. boron doping and secondary phase formation.

Going into the details, a study on $\mathrm{N}$ doped GO QDs has revealed vital findings where nitrogen atom creates an intermediate state (Fig. 8). ${ }^{129}$ Note that in ref. 129 the authors refer GO QDs as graphene QDs while significant quantity of oxygen is evidenced from XPS and EELS. For a suitable illumination, the following transitions are possible, where the wavelength equivalent is given in the brackets for each of them. $6.1 \mathrm{eV}$ : $\pi \rightarrow \pi^{*}$ of $\mathrm{C}=\mathrm{C}(202 \mathrm{~nm}), 4.6 \mathrm{eV}: \pi \rightarrow \pi^{*}$ of $\mathrm{C}=\mathrm{N}(274 \mathrm{~nm})$ and $4.1 \mathrm{eV}: \pi \rightarrow \pi^{*} \mathrm{C}=\mathrm{O}(302 \mathrm{~nm})$, see Fig. 8 .

Tang et al. ${ }^{129}$ suggested two methods of recombination of excited $e-h$ pairs. (i) Direct recombination after vibration relaxation, producing fluorescence and (ii) $\mathrm{C} \pi^{*} \rightarrow \mathrm{N} \pi^{*}$ and

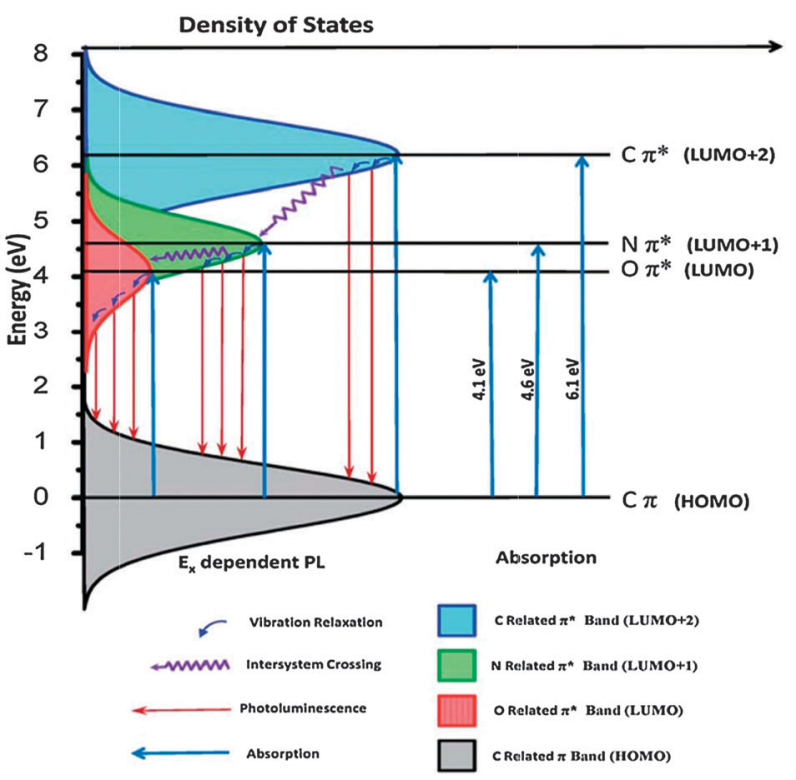

Fig. 8 A schematic diagram illustrating the energy levels of the nitrogen doped GO QDs. Reproduced with permission from ref. 129. 
$\mathrm{N} \pi^{*} \rightarrow \mathrm{O} \pi^{*}$, followed by vibration relaxation and finally radiative recombination. Process (ii) occurs because of the nitrogen doping via intersystem crossing. ${ }^{129}$ The interpretation of emission from GO is based on the involvement of oxygeneous functional groups in contrast to $\mathrm{sp}^{2}$ localization. Significant enhancement of blue emission was achieved after doping rGO with nitrogen (2.3-4.7 at\%) via thermal annealing in the presence of ammonia gas for different time periods. ${ }^{99}$ During this process, formation of graphitic carbon nitride $\left(\mathrm{C}_{3} \mathrm{~N}_{4}\right)$ in $\alpha$ and $\beta$ phases was also detected. These phases impose significant changes in the emission and electronic properties. The emission mechanism explained in ref. 99 is based on localization of $\mathrm{sp}^{2}$ clusters. $^{36}$

A typical emission spectrum from boron doped GO is shown in Fig. 9. The emission is attributed to the recombination of $e-h$ pairs within the electronic band gaps of $\mathrm{sp}^{2}$ clusters ${ }^{71,93,94}$ including the effects from size, shape and fraction. ${ }^{36}$ The fluorescence spectrum of as synthesized GO consists of three components centered at 520,716, and $827 \mathrm{~nm}$, while the size of $\mathrm{sp}^{2}$ clusters increased to $6.90 \mathrm{~nm}$ after B-doping. Despite the increase in the $\mathrm{sp}^{2}$ cluster size the green emission peak is blue shifted (to $494 \mathrm{~nm}$ ) as compared to that of annealed GO with a decrease in its intensity. The second peak at $\sim 636 \mathrm{~nm}$ is attributed to the boron carbide phase $\left(\mathrm{B}_{4.23} \mathrm{C}\right.$ emits $\sim 795 \mathrm{~nm}$ ref. $132, \mathrm{~B}_{4.3} \mathrm{C}, \mathrm{B}_{6.5} \mathrm{C}$, and $\mathrm{B}_{10} \mathrm{C}$ emit $>595 \mathrm{~nm}$ ref. 133).

\subsection{Covalent modification}

In the previous section we have seen substitutional doping and its effects on emission properties of GO and rGO. In this section, we will see the variations in optical properties when GO or rGO were covalently functionalized with various moieties. The covalent functionalization is facilitated through the surface functional groups of GO or rGO. In this direction, researchers have studied considerable types of modifications aiming at various applications ${ }^{81,134}$ including nonlinear optical properties. ${ }^{135-137}$ Typical modifications are surface passivation of the reactive sites, ${ }^{89}$ chemical bonding with fluorescent ions ${ }^{90}$ etc. The covalent modification has various advantages such as improved solubility in intermediate organic solvents, coupling with other functional materials where the spacer length can be tuned and the quantity of loading can be increased. In a typical example, the functionalization can take loading as high as $5 \mathrm{wt} \%$ of dye. ${ }^{79}$

In an approach shown recently ${ }^{79}$ the covalent attachment to GO does not alter the absorption and emission properties of the dye. On the other hand the $\mathrm{pH}$ sensing capability is achieved through the amidic group via reversible protonation. GO layers were functionalized with azo-pyridine ${ }^{81}$ at an interlayer separation of $0.9 \mathrm{~nm}$ showing a bright blue emission via excited ESIPT. The fluorescence spectrum $\left(\lambda_{\mathrm{ex}}=416 \mathrm{~nm}\right)$ of the GO solution (QY $=$ $0.03 \%$ ) depicted a broad peak at $\sim 560 \mathrm{~nm} .^{138-140}$ This peak is blue shifted to $470 \mathrm{~nm}$ for the GO-azopyridine (QY $=8 \%$ ) and the intensity increases $400 \%$ with respect to GO. Basically, functionalization not only creates but also enhances the luminescent centers in the composite. The enhanced optical emission is because of ESIPT between the - $\mathrm{OH}$ group (alpha) of the phenol moiety and the azo group. This is similar to substituted hydroxyl benzaldehydes
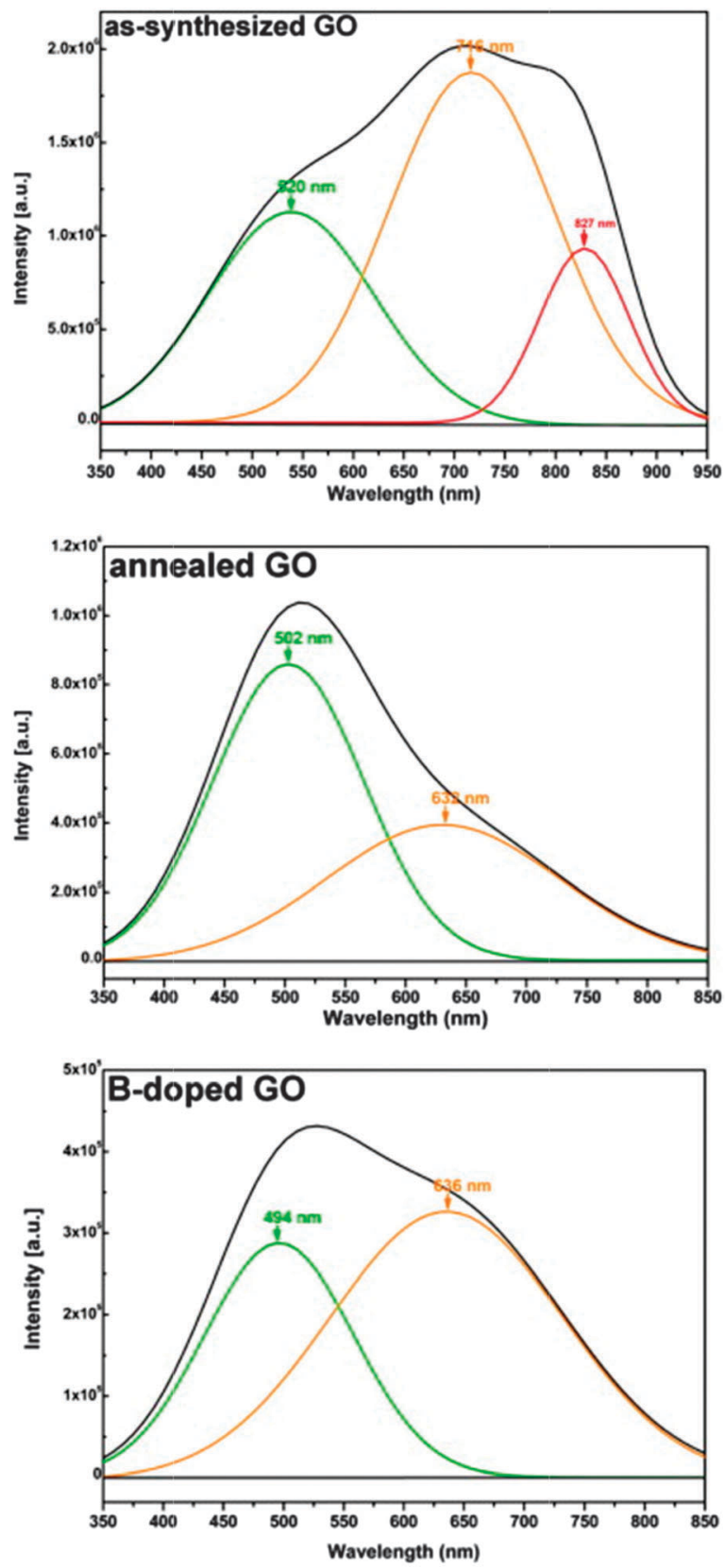

Fig. 9 Emission spectra of as-synthesized GO, annealed GO, and B-doped GO. Reproduced with permission from ref. 128.

where the emission is due to the keto $(\mathrm{H})$ form and the enol-Azo form of ESIPT. ${ }^{141}$

The covalent functionalization of GO with anthryl moieties is interesting. ${ }^{134}$ The emission properties of 2-aminoanthracene (pale yellow under daylight, cyan (491 nm) under $365 \mathrm{~nm}$ ) were significantly changed when functionalized with GO (dark red under daylight, blue (400 nm) under $365 \mathrm{~nm}$ ). This leaves us with a shift of $\sim 91 \mathrm{~nm}$. Such a large shift is simply attributed to the interaction between the anthryl moieties and GO, however, the shift is almost absent when the components are physically mixed. Hence the interaction between $\pi$-orbitals is insignificant for the shift. Hence a deeper investigation is required to 


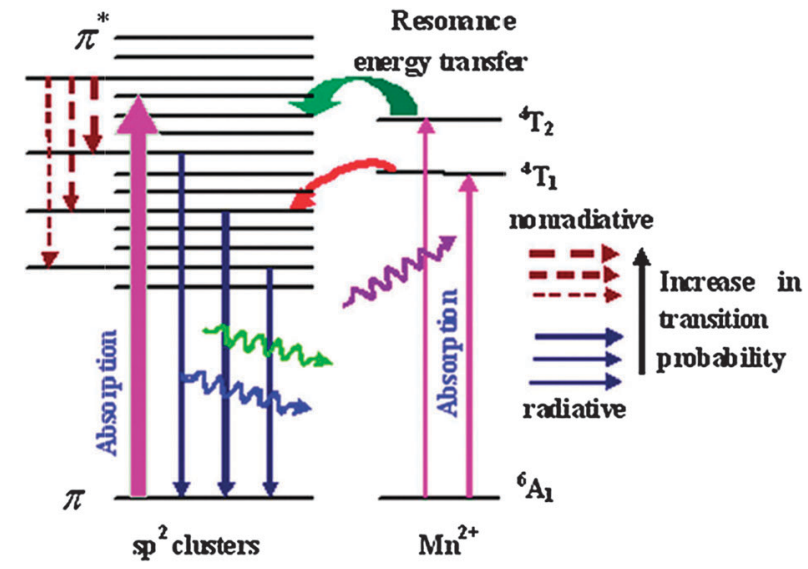

Fig. 10 Schematic mechanism of fluorescence from the $\mathrm{Mn}^{2+}$-bonded rGO where solid and dotted lines represent the radiative and nonradiative relaxation processes, respectively. Reproduced with permission from ref. 90 .

explain how an unconjugated covalent bond causes such a significant shift.

Chemical bonding with fluorescent ions such as $\mathrm{Mn}^{2+}$ has shown interesting results. ${ }^{90}$ The authors attribute emission from rGO to the $\pi-\pi^{*}$ transitions due to localization while resonance energy transfer takes place from the $\mathrm{Mn}^{2+}$ ion to $\pi^{*}$ states of rGO (Fig. 10). ${ }^{90}$ In this hybrid, Mn ions are bonded to the carboxyl groups of rGO which places the ion in the close proximity of $\mathrm{sp}^{2}$ cluster. Finally the authors note that the emission from GO is enhanced.

\section{Nonlinear optical response}

In principle GO can be a more suitable material for optical limiting applications than Gra because of the tunable energy gap. It would be appropriate to briefly describe some unique nonlinear optical features depicted by GO. By definition, a nonlinear property is that the transmission decreases with increasing light intensity (good linear absorption at low input levels). This feature is extremely useful for eye protection where a broadband (visible to IR if possible) optical limiter is demanded. The nonlinear response of GO is different from that of the other carbon allotropes while similar to organic materials. ${ }^{142}$ In the case of GO, for picosecond pulses two-photon absorption is predominant, while for nanosecond pulses excited state nonlinearities play a vital role. ${ }^{142}$ Although Gra is considered for such applications, ${ }^{6} \mathrm{GO}$ has its own advantages such as $2 \mathrm{D}$ nature and more importantly its functionalizability. The functionalizability allows covalent bonding of organic dye molecules (see Section 3.6) or other complementary optical materials and composites. ${ }^{143,144}$ Interestingly, GO depicts better optical limiting response than fullerene $\left(\mathrm{C}_{60}\right)$ as shown by various groups. ${ }^{145,146}$ Experimentally it is evidenced that covalent functionalization with $\mathrm{C}_{60},{ }^{135}$ porphyrin, ${ }^{135,136}$ or oligothiophene ${ }^{137}$ improves the nonlinear optical performance in the nanosecond region. These studies suggest that the hybrid materials have better nonlinear absorption via photoinduced electron or energy transfer. Fluorinated GO has shown higher nonlinear absorption, nonlinear scattering and optical limiting threshold which are about an order of magnitude better than GO. ${ }^{147}$

\section{Ionic interactions}

In the earlier sections we have seen that the functional groups on the GO may be one of the causes for the emission where they open the band gap of graphite. These functional groups are mainly oxygen-contained, which are prone to external interferences such as ions (cations and/or anions). In the following we will discuss the emission dependent on $\mathrm{H}^{+}(\mathrm{pH})$ and other ionic species in two different subsections.

\section{1 pH dependent optical emission}

Essentially, the Fermi level of GO is shifted depending on the $\mathrm{pH}$ values where the electronic structure of GO is manipulated. As a result different emission colors are noticed. ${ }^{69}$ Note that this is in contrast to the GO-azo pyridine composite, where the increased symmetry of the $\pi-\pi^{*}$ state decreases the FranckCondon factor. Consequently radiationless decay is decreased, thereby the fluorescence from such composites gets brighter with decrease in $\mathrm{pH} .{ }^{81}$ Blue fluorescence from GO QDs is found to be $\mathrm{pH}$-dependent where they were derived from cleaving CNT possessing zigzag sites. ${ }^{37}$ The suggested mechanism hinges on the protonation of the emissive zigzag sites where their ground state is $\sigma^{1} \pi^{1}$. The fluorescence can be recovered when deprotonated (alkaline conditions). Multicolour fluorescent GO was synthesized by cleaving CNT upon oxidation ${ }^{69}$ while the fluorescence depicted bathochromic shift ${ }^{148}$ which was attributed to deprotonation of $-\mathrm{OH}$ and $-\mathrm{COOH}$ groups. ${ }^{149,150}$ It is also notable that ionic-liquidassisted electrochemical exfoliation showed similar results. ${ }^{151}$ The intensity of the emission from azo-pyridine functionalized $\mathrm{GO}^{81}$ can be controlled by adjusting the $\mathrm{pH}$ value. In this case the radiatiave surface defects are passivated. ${ }^{149,152}$ The intensity changes are because of the protonation and deprotonation of the functional groups which may cause electrostatic doping (i.e. shift of the Fermi level as seen in the case of carboxylate SWNTs ${ }^{153}$ ). Interestingly, this is in contrast to the fluorescence of GO QDs with change in $\mathrm{pH}$ where the intensity of fluorescence decreases with decreasing $\mathrm{pH}$ (13 to 1 ). ${ }^{37}$ In a study by Peng et al. ${ }^{112}$ the GO QDs emit strong fluorescence under alkaline conditions. While under acidic conditions the fluorescence is quenched because of the protonated free zigzag sites.

\subsection{Other ionic species}

Since GO QDs consists of oxygen containing functional groups they can act as a sensing platform when interacting with ions, $c f$. protons, in the case of $\mathrm{pH}$. The variation in the emission intensity is related to the molecular interaction. The quenching occurs because of inner filter effects, creation of non-radiative paths, electron transfer process and ion binding interactions. ${ }^{154}$ In this section we will see two types of effects because of ionic interactions. (i) The quenching of fluorescence by itself in the 
presence of guest ions and (ii) quenching of the fluorescence of other materials.

Generally quenching of fluorescence of the host in the presence of guest ions takes place through collisional or dynamic quenching. The Stern-Volmer equation ${ }^{155}$ describes the dynamic and collisional quenching via $F_{0} / F=\tau_{0} / \tau=1+$ $k_{\mathrm{q}} \tau_{0}[Q]$, where $F_{0}$ and $F$ are the fluorescence intensities before and after the arrival of guests, respectively. $k_{\mathrm{q}}$ is the rate constant of dynamic (collisional) quenching; $\tau_{0}$ and $\tau$ are lifetimes of fluorophore before and after the arrival of guest ions, respectively; and $[Q]$ is the concentration of the guest ions in the solution. In the context of static quenching, a nonfluorescent complex forms between the host and guest and as a result the life-time of the fluorophore is unperturbed, i.e. $\tau_{0} / \tau=1$. Now, the $k_{\mathrm{q}} \tau_{0}$ is called as association constant. ${ }^{155}$

GO QDs were employed as selective ion sensing materials where the quenching of fluorescence was observed (inversely proportional) under the influence of $\mathrm{Cu}^{2+} \cdot{ }^{28}$ The intensity was linearly decreasing within the range of $0-15 \mu \mathrm{M}$ of $\mathrm{Cu}^{2+}$ ions with a maximum detection limit of $0.226 \mu \mathrm{M}$. Authors also suggest that the quenching mechanism is predominantly static in nature as described by Stern-Volmer equation. ${ }^{28}$ The interaction with $\mathrm{P}^{-}$and $\mathrm{P}^{+}$was studied with $\mathrm{Au}$ NPs and GO separately by Mamidala et al. ${ }^{91}$ Emission from various combinations were shown in Fig. 11a and b. We can see the quenching of emission at $640 \mathrm{~nm}$ from $\mathrm{GO}+\mathrm{P}^{+}$complex in contrast to $\mathrm{GO}+\mathrm{P}^{-}$complex. This indicates that the interacting donor-acceptor complexes are formed between opposite charges. The quenching is attributed to photoinduced electron or/and energy transfer. ${ }^{156}$ This interaction is also reflected in the fluorescence lifetimes (Fig. 11c and d and Table 1 for the time scales). On the other hand, in the case of positively charged picket-fence porphyrin the interaction is attributed to the $\pi-\pi$ type. $^{157}$
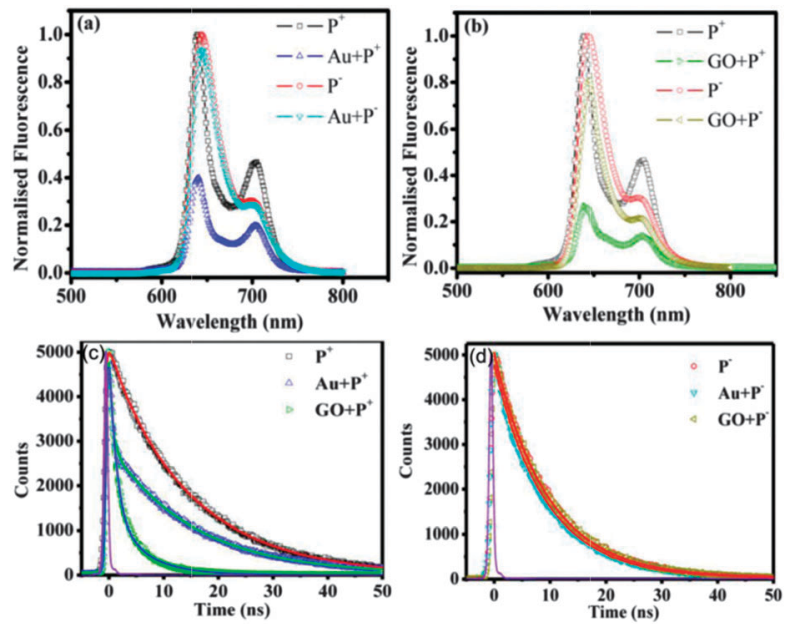

Fig. 11 Spectra of $\mathrm{P}^{+}, \mathrm{P}^{-}, \mathrm{Au}+\mathrm{P}^{+}, \mathrm{Au}+\mathrm{P}^{-}, \mathrm{GO}+\mathrm{P}^{+}$or $\mathrm{GO}+\mathrm{P}^{-}$in water dispersion (a) and (b) fluorescence, $\lambda_{\mathrm{ex}}=430 \mathrm{~nm}$. (c) and (d) decay curves. The instrument response function is shown in violet color trace. Figure reproduced with permission from ref. 91
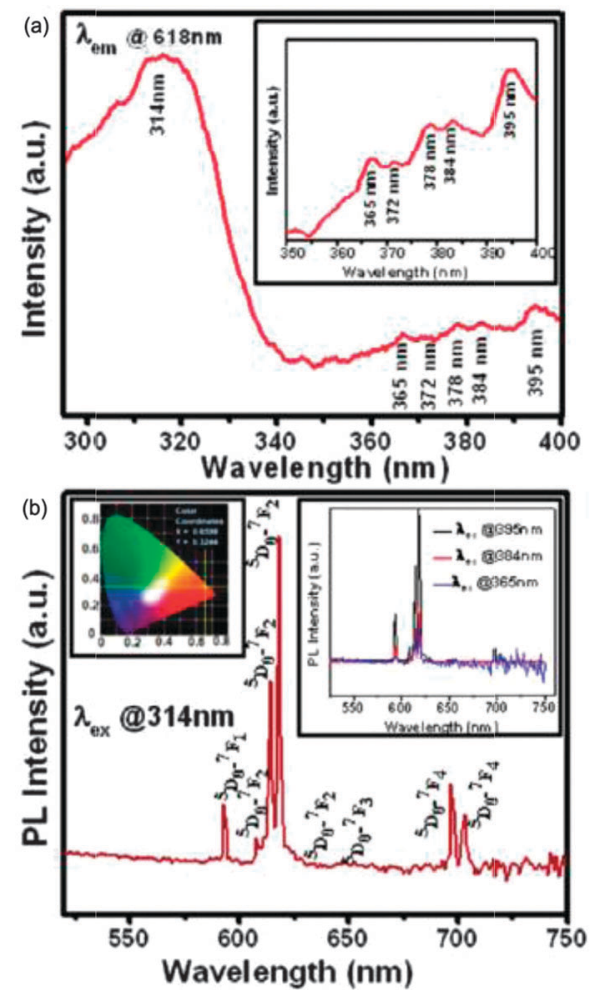

Fig. 12 (a) Fluorescence excitation spectrum of the $\mathrm{rGO}$ and $\mathrm{Eu}^{3+}$ complex, inset is the $350-400 \mathrm{~nm}$ region, and (b) fluorescence spectrum $\left(\lambda_{\mathrm{ex}}=314 \mathrm{~nm}\right)$. The right inset shows the other three distinct emission spectra at different $\lambda_{\mathrm{ex}}$ and the left inset shows the color coordinates $(x=$ $\sim 0.66$ and $y=\sim 0.32$ ). Reproduced with permission from ref. 158 .

Previously, interaction of GO with charged porphyrin ${ }^{91}$ has been discussed; similarly $\mathrm{Eu}^{3+}$ ions are also a subject of investigation against the fluorescence from rGO (Fig. 12). ${ }^{158}$ In this study, the authors referred to rGO as graphene as it contains very low percentage of oxygen. Nevertheless, the complexation requires oxygen functionalities on graphene, hence, we will be referring to this as rGO instead of graphene. This complex is shown to quench the fluorescence of Rhodamine-B dye while the complex of $\mathrm{Eu}^{3+} / \mathrm{rGO}$ is active $\left(\lambda_{\mathrm{ex}}=314 \mathrm{~nm}, \lambda_{\mathrm{em}}=\right.$ 614 and $618 \mathrm{~nm})$. Note that the various oxygeneous functional groups on rGO spatially distributed around the $\mathrm{Eu}^{3+}$ ion should be at low symmetry sites. ${ }^{159}$ This is in contrast to an earlier explanation, ${ }^{90}$ where an energy transfer takes place from $\mathrm{Mn}^{2+}$ to the localized states of $\mathrm{sp}^{2}$ on rGO. In this case the involvement of oxygen containing functional groups can be avoided, despite the covalent bond between rGO and $\mathrm{Mn}^{2+}$ (see Section 3.6 and Fig. 10). Also see anthryl functionalized GO and its emission properties ${ }^{134}$ in Section 3.6. In the PLE spectrum (Fig. 12) the interacting oxygen functionalities and $\mathrm{Eu}^{3+}$ have shown a strong band at $314 \mathrm{~nm}^{160,161}$ while the other five peaks are attributed to $\mathrm{f}-\mathrm{f}$ transitions of the $\mathrm{Eu}^{3+}$ ions. The authors suggest triple-exponential decay (average lifetime $\sim 391.13 \mu \mathrm{s}$ ) due to the differences in the ligand environments in the rGO around $\mathrm{Eu}^{3+}$. The combination of GO is not limited to $\mathrm{Eu}^{3+}$ but extends to europium oxide. ${ }^{162}$ 


\section{6. $\pi-\pi$ type interactions}

Moving onto the combinations with organic semiconductors, Yang et $a .^{163}$ studied fluorescence from the GO-P3HT nanocomposite heterostructure and suggested a $\pi-\pi$ interaction between the two components. ${ }^{164-166}$ In this heterostructure P3HT chains are attached to rGO while the former coats a thin-layer on the latter. Later in 2012, PDS and pump-probe techniques were employed on the GO-P3HT layer-to-layer hybrid, and the results support the earlier argument of $\pi-\pi$ interaction (Fig. 13). ${ }^{92}$ In the solution phase, the normalized PL spectra of P3HT, GO-P3HT and rGO-P3HT are of comparable intensity (Fig. 13a), with small differences in the range 540$600 \mathrm{~nm}$, see inset. In contrast to this, in the solid phase the presence of GO or rGO has significantly quenched the emission from P3HT (Fig. 13b) via $\pi-\pi$ (weak Coulombic) interactions. See Table 1 and Fig. 13c for decay times and measurements, respectively. Furthermore the transient response studies (650 nm, Fig. 13d) indicated that the GO-P3HT composite did not show any stimulated emission. However a photoinduced absorption signal with two decay times ( $\tau=1.4$ and $38.5 \mathrm{ps})$ is observed in contrast to pure P3HT which depicted stimulated emission. As a result, an ultrafast charge dissociation of P3HT excitons ${ }^{167}$ takes place at the interface and charge pairs are injected into GO as fast as $1.4 \mathrm{ps}$. In the case of rGO-P3HT the electrons generated in $\mathrm{P} 3 \mathrm{HT}$ are injected rapidly into rGO. In this context both GO and rGO are very useful in solar cells where fast transfer of photogenerated charge is the primary objective. ${ }^{168}$ In the case of covalent functionalization between $\mathrm{P} 3 \mathrm{HT}$ and $\mathrm{GO}^{169}$ the overall fluorescence quenching includes dynamic quenching and forms a non-fluorescent ground-state complex. ${ }^{169}$ Also this $\pi-\pi$ interaction blue shifted $(\sim 4 \mathrm{~nm})$ the absorption maximum of P3HT. It would be more conclusive if the XRD patterns were investigated on solid samples, where the consequence of $\pi-\pi$
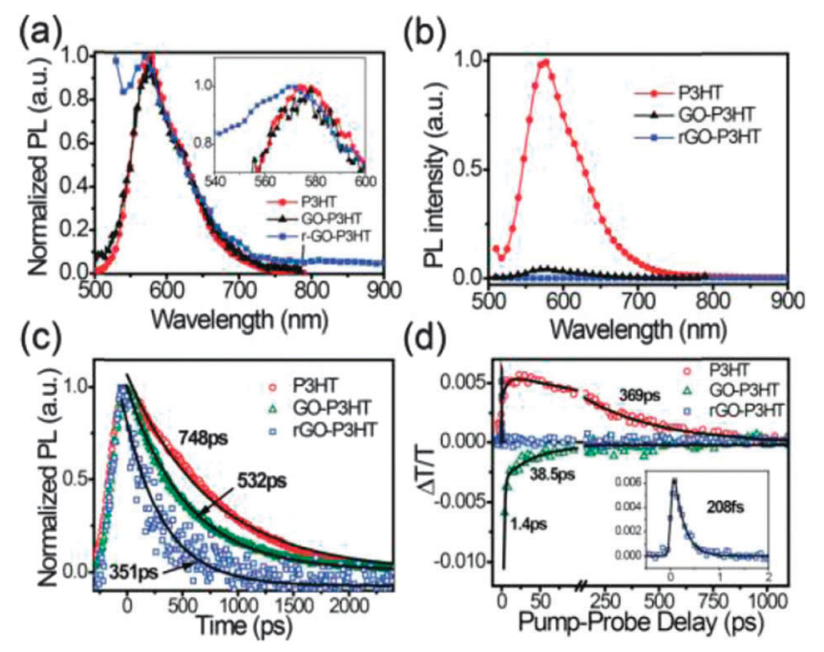

Fig. 13 (a) Normalized fluorescence spectra, (b) fluorescence intensity while P3HT is $0.1 \mathrm{mg} \mathrm{mL}^{-1}$, (c) TCSPC decay curves and (d) relative changes in transmission for varying pump $\left(10 \mu \mathrm{J} \mathrm{cm}{ }^{-2}\right)$-probe delays; $\lambda_{\mathrm{ex}}=400 \mathrm{~nm}$ and $\lambda_{\mathrm{em}}=650 \mathrm{~nm}$. The inset shows the magnified spectrum of rGO-P3HT in the first 2 ps. All cases are dispersion in $\mathrm{CHCl}_{3}$. Reproduced with permission from ref. 92 . interaction and layer formation can be understood rather precisely via (002) interplanar spacing of GO.

It is important to note that the case with PANI is not similar to P3HT or even inorganic semiconductors. When graphene is combined with PANI either through in situ polymerization or mixing $^{170}$ the emission properties of PANI were preserved suggesting an inappropriate band alignment and possible $\pi-\pi$ interaction.

The fluorescence from rGO and its decay life time were enhanced with $\mathrm{Rb}^{70}$ through non-covalent bonding. Apart from preserving the native features of rGO such as excitation dependent fluorescence, a slight shift in the peak position is observed. From the fluorescence decay (Table 1) it is suggested that the shorter component has higher contribution $(\sim 84 \%) .{ }^{58,87}$ Another study on $\pi-\pi$ interactions of rGO with positively charged picket-fence porphyrin ${ }^{157}$ suggested a quenching of fluorescence from porphyrin under the influence of rGO.

\section{Heterointerfaces}

\subsection{Photovoltaics}

GO and rGO are proven to be potential for photovoltaic applications. For example, the hole transport property of PEDOT:PSS can be improved with the addition of GO at a suitable concentration. ${ }^{9}$ Furthermore such combinations can yield a band gap larger than $1.1 \mathrm{eV}$ for $10-15 \mathrm{wt} \%$ of GO, while the carrier transport property is majorly determined by the fine structure of host PEDOT:PSS. ${ }^{171}$ At a certain concentration, GO in dye-sensitized solar cells acts as an electron collector and transporter resulting in an enhanced photovoltaic performance. ${ }^{172}$ Moreover it also improves the transfer of electrons from the films to the FTO substrate. ${ }^{173}$ In contrast to this, partially reduced GO is employed as an active layer and rGO is employed as electron and hole collecting layers. This symmetric device configuration is shown in Fig. 14a. The device has depicted a $V_{\text {oc }}$ of 0.017-0.014 V. However the authors in ref. 174 did not present any fluorescence data from partially reduced GO and rGO in the case if there is any. ${ }^{15,36,58}$ Despite this, this study is remarkable where it employs rGO as an active material in the device. Although the fluorescence from rGO is debatable, however, the energetic states and their alignment can be deduced by fabricating pn-junctions. ${ }^{18}$ Such studies can unveil the information about charge generation and subsequent separation. Composite HJs were studied (a)

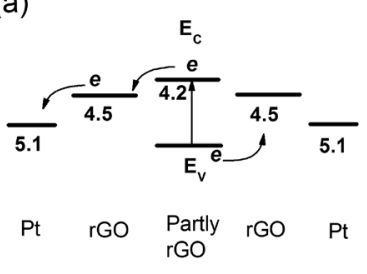

(b)

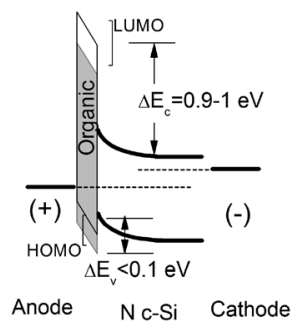

Fig. 14 (a) Schematic energy level diagram of $\mathrm{rGO}$ based solar cell (b) band diagram of the crystalline silicon ( $\mathrm{N} \mathrm{C}$-Si) and PEDOT:PSS/GO composite junction under small FB. Figure redrawn based on ref. 171 and 174. 
for electrical characteristics where they can be integrated into the well established silicon devices. ${ }^{171}$ The authors have studied carrier transport in crystalline-Si (100) (c-Si)/conductive PEDOT:PSS composite HJs. ${ }^{171}$ See Fig. 14b for the band diagram under a small FB. The results suggest that the carrier transport mechanism is changed from diffusion to the space-charge recombination with the increase of GO content in PEDOT:PSS. Upon introducing GO in PEDOT:PSS, apart from the improvement in the ideality factor (GO-PEDOT:PSS-2.91 PEDOT:PSS-1.12) the efficiency of the device is enhanced. ${ }^{171}$ The cell characteristics are $\eta=\sim 10.3 \%, J_{\mathrm{sc}}=$ $28.9 \mathrm{~mA} \mathrm{~cm}{ }^{-1},{ }^{2} V_{\mathrm{oc}}=0.548 \mathrm{~V}$ and $\mathrm{FF}=0.675$ at a $\mathrm{GO}$ content of $12.5 \mathrm{wt} \%$ with diffusion and recombination in the space-charge region. Improvements in charge extraction efficiency and reduced charge recombination were observed by inserting the $\mathrm{rGO}-\mathrm{TiO}_{2}$ composite layer as an optical spacer between the active layer and $\mathrm{Al}$ electrode. ${ }^{175}$ This interfacial layer blocks the holes as well. As a result the $\mathrm{PCE}$ is $\sim 4.18 \%$ and $\sim 5.33 \%$ for $\mathrm{TiO}_{2}$ and the $\mathrm{rGO}-\mathrm{TiO}_{2}$ interfacial layer, respectively where a similar structure without the interfacial layer has shown a value of $\sim 3.26 \%$. It is obvious that defects of GO or rGO influence the device performance. However, in an interesting study by Chang et $a .^{176}$ the defects and atomic structure are controlled yielding well regulated infrared PR (responsivity of $\sim 0.7 \mathrm{~A} \mathrm{~W}^{-1}$ ) in rGO phototransistors. This study evidenced that the PR is mostly dependent on oxygenous defects. Furthermore external quantum efficiency of $\sim 97 \%$ and no PR degradation even after 1000 bending cycles are significant.

\subsection{Nanocomposites}

Nanocomposites are very potential materials in scientific and technological applications in which rGO or GO is employed extensively. The host matrices are inorganic or organic in nature depending on the type of application. Inorganic matrices can be CdSe nanoparticles, ${ }^{177} \mathrm{ZnO}$ @ZnS hollow dumbbells, ${ }^{29}$ zinc (hydr) oxide, ${ }^{178} \mathrm{TiO}_{2}{ }^{77,179,180}$ Fe-doped $\mathrm{TiO}_{2}$ nanowires, ${ }^{181}$ noble metal doped $\mathrm{TiO}_{2}{ }^{182} \mathrm{ZnO},{ }^{180,183,184} \mathrm{Ag} / \mathrm{ZnO},{ }^{185,186} \mathrm{ZnS},{ }^{187} \mathrm{CdS},{ }^{188-191}$ $\mathrm{Ta}_{2} \mathrm{O}_{5},{ }^{180} \mathrm{CdSe},{ }^{177,192} \mathrm{CdTe},{ }^{193} \mathrm{Ag}_{2} \mathrm{Se}^{194}$ etc. Examples of organic components include PANI, ${ }^{170,195}$ P3HT, ${ }^{163}$ methylcellulose ${ }^{143}$ etc. In this section we will focus on the optical properties of these material combinations in the context of charge transfer, where the relative position of HOMO and LUMO levels play a crucial role.

When CdSe NPs were composited with $\mathrm{rGO}^{177}$ the PL from CdSe is observed to decrease apart from an enhancement in the PR. This indicates that the photoinduced carriers from the CdSe NPs can be transferred to the rGO effectively. A recent investigation $^{196}$ on ZnO and GO QDs presents important insights in the emission (Fig. 15a) from a composite with an application in LEDs. The MO levels, DOS for pristine and G-O with an epoxy bond ( $\left.\mathrm{G}-\mathrm{O}_{\text {epoxy }}\right)$ including the oxygen PDOS are shown in Fig. 15b. The results indicate that there are significant orbital hybridizations after the chemical bond with the oxygen atom. The mechanism of emission is shown in Fig. 15c. Under illumination the photo-excited electrons from the $\mathrm{O} 2 \mathrm{p}$ of the $\mathrm{ZnO}$ are transferred to the unoccupied states of $\mathrm{G}-\mathrm{O}_{\text {epoxy. Then }}$ these electrons recombine with the holes in $\mathrm{VB}$ of $\mathrm{ZnO}$ creating two additional peaks in the spectrum. Such transitions are determined by the selection rule $(\Delta l= \pm 1)$, i.e. $l=0$ or $l=2$
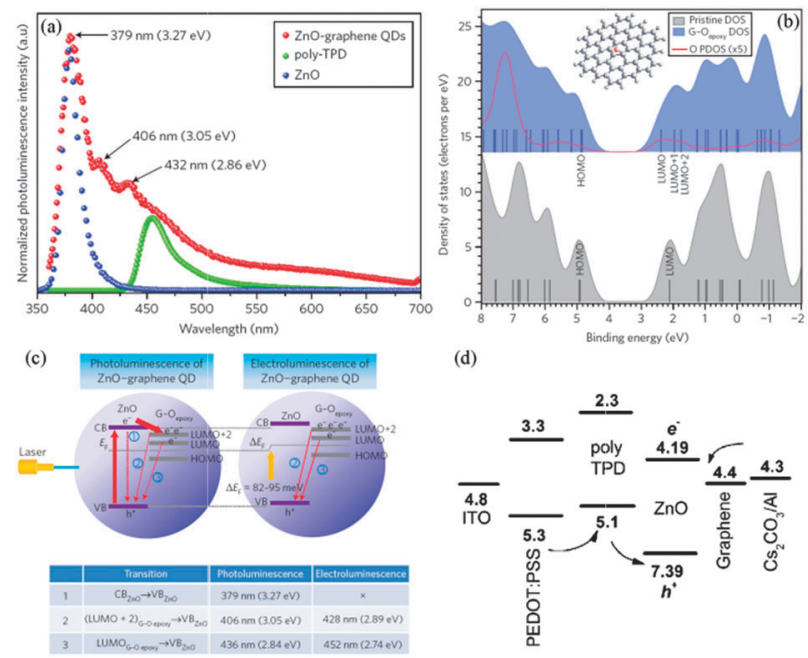

(d)

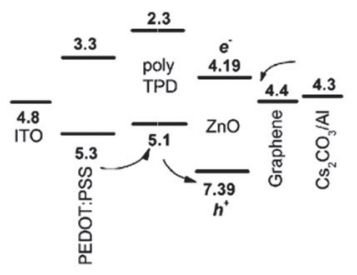

Fig. 15 (a) Emission spectra, (b) DOS of graphene and the $\mathrm{G}-\mathrm{O}_{\text {epoxy }}$ model. MO energy is indicated with vertical bars in each calculated DOS. Inset: G-O epoxy model, (c) PL and EL transition scheme for $\mathrm{ZnO}-\mathrm{GO}$ QDs, (d) band alignment of various components in the LED. Parts (a)-(c) are reproduced with permission from ref. 196 and part (d) is redrawn based on ref. 196 .

electrons can recombine with $\mathrm{O} 2 \mathrm{p}(l=1)$. Contextually, DFT results suggest that only p orbitals contribute to the LUMO level of pristine graphene and hence no transitions as $l=1$ and the un-hybridized LUMO level splits into three levels with oxygen attachment (LUMO, LUMO + 1 and LUMO + 2). See Fig. 15c for various allowed transitions. The emission from $\mathrm{ZnO}-\mathrm{GO}$ QDs is deconvoluted into four Lorentzian peaks centred at 379 (band to band), 406 (LUMO + 2 in G-O epoxy to VB of ZnO), 436 (LUMO in $\mathrm{G}-\mathrm{O}_{\text {epoxy }}$ to $\mathrm{VB}$ of $\left.\mathrm{ZnO}\right)$ and $550 \mathrm{~nm}\left(V_{\mathrm{O}}\right.$ or $\left.\mathrm{Zn}_{i}\right)$, according to the authors' attribution.

Optical and electrochemical properties of $\mathrm{ZnO}$ nanowires/ GO heterostructures reveal that GO can suppress surface states of $\mathrm{ZnO}$ enhancing the UV-emission of $\mathrm{ZnO}{ }^{197}$ This enhancement is a balance against the green emission, which is due to $V_{\mathrm{O}} \mathrm{S}$ in $\mathrm{ZnO}$ as widely accepted, ${ }^{16-18}$ also see cross references in ref. 17. There is also a possibility that the electrons are transferred to GO due to the energy level alignment (Fig. 16a). Of course, GO can perhaps passivate the surface ${ }^{198}$ in which case ionized $V_{\mathrm{O}} \mathrm{s}$ can be suppressed, thereby enhancing the UV emission. A similar case can be seen in the literature, ${ }^{199}$ in which the authors compared PL properties of $\mathrm{ZnO}$ nanorods when coated with GO and rGO. Notably, the emission due to interband transition is enhanced when $\mathrm{ZnO}$ nanorods were coated with rGO (Fig. 16a). In another example of the GO-ZnO composite, ${ }^{183}$ the green emission (centered at $\sim 550 \mathrm{~nm}$ ) from $\mathrm{ZnO}$ was blue shifted $(0.15 \mathrm{eV})$ and quenched upon compositing with GO. The authors suggest additional pathways for the subdued emission via interfacial charge transfer from $\mathrm{ZnO}$ to GO. ${ }^{200}$ In ref. 200 the authors show that the PL quenching increases with increasing concentration of GO without any shift in the PL peak position. This might be because of the preparation technique that is used. It is notable that the electrons from the $\mathrm{ZnO}$ were primarily used in the reduction of GO to rGO upon 
(a)

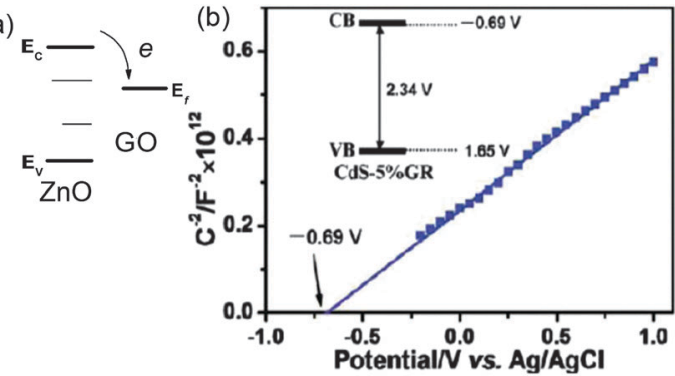

Fig. 16 Schematic diagram of the electron transfer between (a) ZnO NWs and GO films and (b) Mott-Schottky plot for the CdS-5\% rGO nanocomposite in $0.2 \mathrm{M} \mathrm{Na}_{2} \mathrm{SO}_{4}$ aqueous solution $(\mathrm{pH}=6.8$ ), reproduced with permission from ref. 203

irradiation of UV light. In contrast, to ref. 200 Singh et al. ${ }^{183}$ eliminated the electron transfer from $\mathrm{ZnO}$ to GO via modifying the preparation method. It is also suggested that the interaction is similar to $\mathrm{SnO}_{2}-\mathrm{CNT}$ or ZnO-SWNT composites. ${ }^{201}$ Although the suppression of $V_{\mathrm{O}} \mathrm{S}$ is explicit the mechanism behind such passivation and the creation of additional pathways should be studied further. On the other hand the fluorescence from GO is also seen to quench ${ }^{202}$ when combined with $\mathrm{ZnO}^{184}$

Mott-Schottky plots (Fig. 16b) provide information about the feasibility of transfer of photogenerated electrons to rGO. ${ }^{203}$ In the case of $\mathrm{CdS}$ nanoparticles, it is thermodynamically permissible for the absorbed $\mathrm{O}_{2}$ to produce superoxide radicals $\left({ }^{\bullet} \mathrm{O}_{2}{ }^{-}\right)$under visible light illumination. The photoinduced electrons are transferred to rGO delaying the recombination process. ${ }^{203}$ Similar to the earlier cases the PL intensity from CdS is subdued. ${ }^{190,204}$ By considering the energetic locations ${ }^{205}$ of CdS $\left(\chi_{\mathrm{CdS}}=4.00 \mathrm{eV}\right)$ and $\mathrm{rGO}\left(E_{\mathrm{F}}=4.42 \mathrm{eV}\right)$, under suitable illumination electron transfer occurs from the $\mathrm{CB}$ of $\mathrm{CdS}$ to $\mathrm{rGO}$ and hence the emission is quenched (inset of Fig. 16b).

The fluorescent spectra from GO grafted CdTe (exciton band at $520 \mathrm{~nm}$ ) are shown in Fig. $17 .{ }^{193}$ The emission is centred at $\sim 540 \mathrm{~nm}$ under $365 \mathrm{~nm}$ illumination. In the case of $\mathrm{GO}-\mathrm{Cl}$, the sample has shown some visible fluorescence may be due to sulfonyl chlorination of the GO. As seen earlier, although GO is itself fluorescent it can quench the luminescence of other

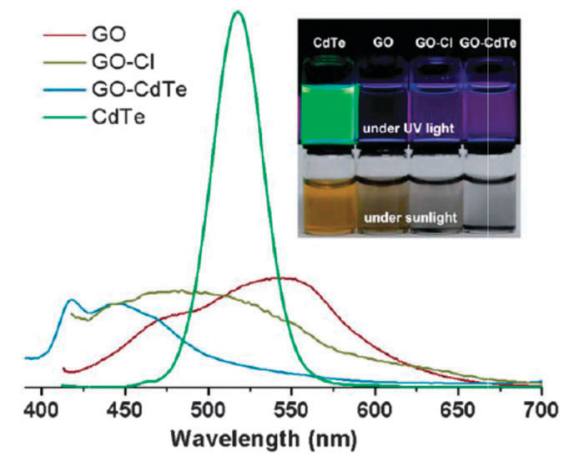

Fig. 17 Fluorescence spectra, $\lambda_{\mathrm{ex}}=365 \mathrm{~nm}$. The inset shows the optical images $\lambda_{\mathrm{ex}}=365 \mathrm{~nm}$ (top) and under $\lambda_{\mathrm{ex}}=$ sunlight (bottom). Reproduced with permission from ref. 193.
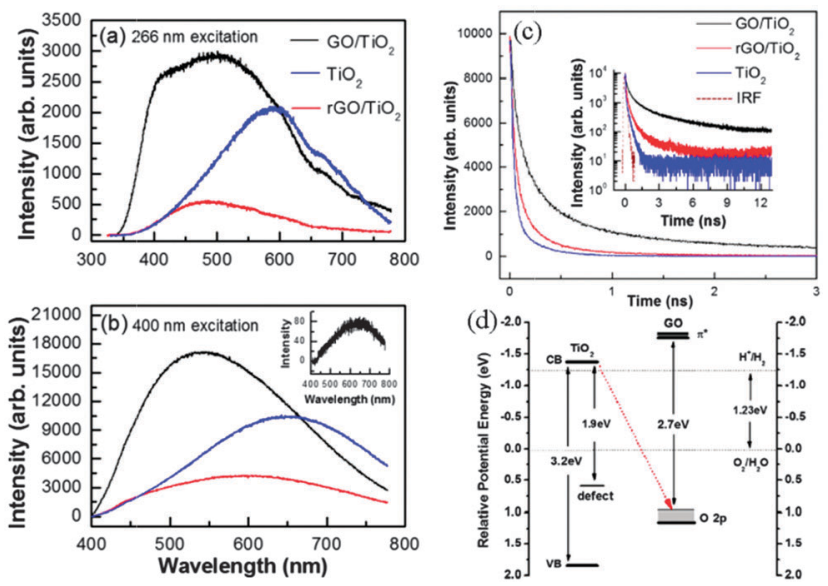

Fig. 18 Emission spectra $\lambda_{\text {ex }}$ is (a) $266 \mathrm{~nm}$ and (b) $400 \mathrm{~nm}$. The inset of (b) shows the fluorescence spectrum of as synthesized GO, $\lambda_{\mathrm{ex}}=400 \mathrm{~nm}$. (c) $\mathrm{PL}$ decay curves, $\lambda_{\mathrm{ex}}=400 \mathrm{~nm}$, inset: 0 to $12 \mathrm{~ns}$ on a log scale. The fluorescence signal was collected over the entire spectrum of each sample. IRF: instrument response function and (d) schematic of band diagram for $\mathrm{TiO}_{2}$ and $\mathrm{GO}$, water oxidation potential is set at $0 \mathrm{eV}$. The dotted arrow red line marks the IOT. Figures are rearranged and reproduced with permission from ref. 77.

materials. ${ }^{188,206,207}$ GO quenched the interband transition (due to fluorescence resonance energy transfer, or nonradiative dipole-dipole coupling between $\mathrm{CdTe}$ and $\mathrm{GO}^{208}$ ) and depicted an emission around $420-450 \mathrm{~nm}$. This is because of the amidation process which creates localized $\mathrm{sp}^{2}$ clusters and structural defects. ${ }^{15,39}$ This is similar to CdSe nanocrystals (cubic and hexagonal) where the PL from CdSe is quenched by rGO. ${ }^{192}$

$\mathrm{TiO}_{2}$ and GO alternative layer structure is studied ${ }^{77}$ for luminescence properties and decay life times. The emission properties and band diagram (ignoring the presence of any defect-related ${ }^{58}$ states) are shown in Fig. 18. For $\mathrm{TiO}_{2}$ the emission peak (at $\sim 600 \mathrm{~nm}$ ) is red shifted $(\sim 650 \mathrm{~nm})$ significantly upon increasing the $\lambda_{\text {ex }}$ which is attributed to vacancy related defects ${ }^{209-211}$ within the band gap. The QY is as low as $<1 \%{ }^{210}$ (Fig. 18c) with a lifetime component that is only a little longer than the $\sim 250$ ps resolution. In the case of $\mathrm{GO} / \mathrm{TiO}_{2}, 550 \mathrm{~nm}$ band is blue shifted (to $\sim 500 \mathrm{~nm}$ ) while the emission is subdued for $\mathrm{rGO} /$ $\mathrm{TiO}_{2}$. Authors attribute this blue-shift to the quenching effect, which is more effective on the longer wavelength side of the PL spectrum of $\mathrm{TiO}_{2}{ }^{211}$ The quenching effect creates non-radiative decay channels and hence a faster PL decay should be noticed. The present decay curves suggest that the fluorescence quenching effect plays a minor role in blue shift. However, the authors attribute the emission to IOT between $\mathrm{TiO}_{2}$ and the localized sp ${ }^{2}$ domains of GO in a charge-separated configuration. From Fig. 18d, the electrons localize in the $\mathrm{CB}$ of $\mathrm{TiO}_{2}$ while the holes can either relax to the defect level or be injected into the $\mathrm{O} 2 \mathrm{p}$ level for both $\lambda_{\mathrm{ex}}$. The optical recombination of electrons from $\mathrm{CB}$ of $\mathrm{TiO}_{2}$ with that of holes in O2p levels of GO is allowed (reduced symmetry at the interface ${ }^{104}$ ). This is seen as the blue-shifted emission (type-II fluorescence ${ }^{105}$ ) for both $\mathrm{GO} / \mathrm{TiO}_{2}$ and $\mathrm{rGO} / \mathrm{TiO}_{2}$ cases. Such recombination occurs due to the intimate contact between the 
components. In the present case the typical distances are $\sim 1.608 \mathrm{~nm}\left(\mathrm{GO} / \mathrm{TiO}_{2}\right)$ and $\sim 1.156 \mathrm{~nm}\left(\mathrm{rGO} / \mathrm{TiO}_{2}\right) .{ }^{212}$ The longer $\mathrm{PL}$ decay time of $\mathrm{GO} / \mathrm{TiO}_{2}$ is attributed to the reduced overlap of electron and hole wave functions. ${ }^{105}$ In the $\mathrm{rGO} / \mathrm{TiO}_{2}$ case the intimacy between $\mathrm{TiO}_{2}$ and rGO increased and hence the interconnectivity of localized $\mathrm{sp}^{2}$ sites and the percentage of zero gap regions is also increased. ${ }^{36}$ This leads to enhanced charge transfer and consequently better quenching of PL with shortened lifetime. ${ }^{36}$ As the oxygen content decreases the O2p level will be lifted up causing a red shift in the PL peak of $\mathrm{rGO} / \mathrm{TiO}_{2}$ relative to that of $\mathrm{GO} / \mathrm{TiO}_{2}$. It is notable that the decay times of $\mathrm{TiO}_{2}$ and $\mathrm{rGO}$ or GO were not in the same order of magnitude and as a result, the composite decay time is an integral effect of their individual characteristics. If one has to consider such an argument, then a way needs to be figured out to resolve the decay constant for each of the components. It is interesting to see that the above interpretation combines the effects of $\mathrm{sp}^{2}$ cluster localization and the involvement of the $\mathrm{O} 2 \mathrm{p}$ level.

The interface between Fe-doped $\mathrm{TiO}_{2}$ and GO enables transfer of electrons from the CB of the semiconductor to GO (Fig. 19a) quenching the overall emission. However Fe-doping enables the creation of $\mathrm{e}-\mathrm{h}$ pairs under visible light illumination. A similar study can be seen on the suppression of PL from $\mathrm{TiO}_{2}$ under the influence of GO. ${ }^{179}$ Furthermore, PL measurements on the GO-ZnS nanocomposite suggest that graphene can be employed to quench the defect level emission. ${ }^{191}$ However, the details of the defect levels and their passivation mechanism were not suggested. Although it is accepted that the defect levels can be passivated variously with polymers or other inorganic coatings, ${ }^{198}$ the energetic location of the defect and its alignment with the bands of GO is a very important factor to consider. We can see other examples in which electron transfer takes place from $\mathrm{TiO}_{2}$ to GO and rGO. ${ }^{182}$ Other inorganic low band gap semiconductors such as $\mathrm{Cu}_{2} \mathrm{O}$ showed a similar effect in terms of transfer of photogenerated charge carriers $\left(\mathrm{Cu}_{2} \mathrm{O} / \mathrm{PA} / \mathrm{rGO}\right.$ and $\left.\mathrm{Cu}_{2} \mathrm{O} / \mathrm{rGO}\right){ }^{187}$ Furthermore the transfer of electrons and/or holes takes place across the interface even if more than one semiconductor is present. It is the case with ZnO@ZnS hollow dumbbells-GO composite; ${ }^{29}$ see the schematic charge transfer process in Fig. 19b.

Fluorescence quenching ability of GO is extended to fluorescein moieties (fluorescein derived silyl ether) ${ }^{213}$ similar to other complexes with organic dyes. ${ }^{136,214}$ The fluorescence quenching is explained based on the band alignments of fluorescein and GO. ${ }^{215}$ This allows the transfer of photogenerted electrons into the CB of GO (Fig. 20). Besides the regeneration of fluorescein is facilitated by $\mathrm{I}_{3}{ }^{-} / \mathrm{I}^{-}$.

(a)

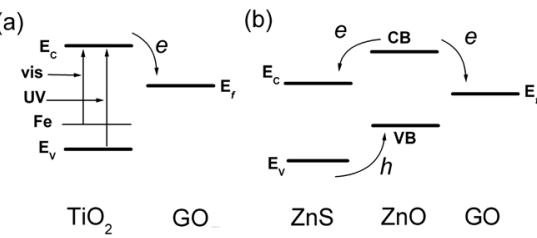

Fig. 19 (a) The interface between Fe-doped $\mathrm{TiO}_{2}$ and $\mathrm{GO}$ (b) the band alignment of $\mathrm{ZnO@ZnS}$ hollow dumbbells-graphene composites. ${ }^{29}$ Figures redrawn based on ref. 29 and 181.

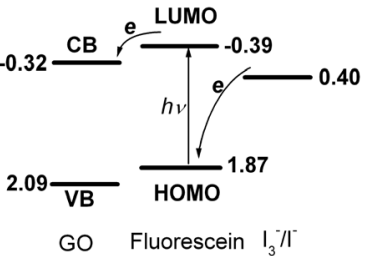

Fig. 20 The energy level diagram illustrating the electron transfer process between $\mathrm{GO}$ and the fluorescein moiety in fluorescein derived silyl ether. Figure redrawn based on ref. 213.

\section{Conclusion and outlook}

In this review we have focused on the optical properties of GO and rGO where the fluorescence properties are explored recently. We have discussed the existing mechanisms and pointed out largely ignored issues such as self-rolling, byproduct formation, concentration, dielectric constant etc. It is important to note that the emission properties of GO depend on the synthesis process. The distribution of various oxygen containing functional groups is completely process dependent. Given the vast amount of the literature, a simple and versatile quantification technique is demanding to quantify the functional groups and their spatial distribution. These two factors play a crucial role in determining the emission properties. Generally, an ensemble of GO sheets is considered for spectral analysis. This ensemble includes various shapes, sizes which are crucial parameters to be evaluated. In case if the sample contains a class of material at low concentration with high QY, then we may observe a predominant emission peak. While that peak wavelength will be interpreted against the majority distribution. In order to avoid such discrepancies one needs to consider studying a single sheet of GO and its fluorescence. Notably, the degree of oxidation and reduction can be employed to tune the emission properties of GO and rGO while a precise control on the relative densities of functional groups still needs to be achieved. For example, during the reduction some of the functional groups are reduced faster than others, although the optical band gap is tunable. The detailed understanding of nanometer- to sub-nanometer-scale structures of GO and rGO can perhaps show new directions for the interpretation of their fluorescence. The plus point is that the oxygen containing functional group on GO and rGO enables further functionalization with other materials. Moreover, it is soluble in a variety of solvents and hence subsequent incorporation into composites is an easy task.

Fluorescence from GO opens new and exciting opportunities for exploration of photonic devices such as LEDs, photodetectors, photovoltaics etc. However, photodetector studies should focus on wavelength selectivity. ${ }^{18}$ Such studies not only give further insight into the energy levels of GO but also shed some light on the fluorescence mechanism in an indirect fashion. The fluorescence from GO and rGO depends on various factors. Despite this, molecular sensing with rGO is quite promising, where its sensitivity to certain chemicals and relatively higher signal-to-noise ratio are worth mentioning. Although there are some in vivo studies, cell imaging etc., for practical applications 
the selectivity to some ions or molecules and recycling require further studies.

The characteristics of GO and rGO are not only determined by the level of oxidation, but also strongly influenced by the distribution of conjugated carbons, holes, vacancies, folds, wrinkles, interfaces of sheets etc. Furthermore one should note the differences in the emission depending on the synthesis process. ${ }^{15,36,58}$ Strong localization of $\mathrm{sp}^{2}$ clusters and involvement of oxygen functional groups in the fluorescence of GO or rGO deserves further attention. If the oxygen functional groups have to be eliminated from the luminescence mechanism, then the $\mathrm{sp}^{2}$ clusters should be localized in a matrix that doesn't contain any oxygen or related functional groups. For example, a complete substitution of oxygen with a suitable element while retaining the lattice constant of oxidized (modified) graphene can be an example target material. Also the average sheet dimensions should not be modified in the process of substitution. This is in clear contrast to a simple reduction of GO. The unique 2D lattices of GO and rGO provide an exciting platform in which various applications and fundamental interests are involved in engineering, physics, chemistry, biology and materials science.

\section{Abbreviations}

$\begin{array}{ll}\text { Materials } & \\ \mathrm{P}^{-} & \text {Negatively charged porphyrin } \\ \mathrm{P}^{+} & \text {Positively charged porphyrin } \\ \mathrm{CNT} & \text { Carbon nanotube } \\ \text { Au NPs } & \text { Au nanoparticles } \\ \text { PEDOT:PSS } & \text { Poly(3,4-ethylenedioxythiophene) polystyrene } \\ & \text { sulfonate } \\ \text { Poly-TPD } & \text { Poly( } N, N^{\prime} \text {-bis(4-butylphenyl)- } N, N^{\prime} \text {-bis(phenyl) } \\ & \text { benzidine) } \\ \text { SLG } & \text { Single layer graphene } \\ \text { PEG } & \text { Polyethylene glycol } \\ \text { PA } & n \text {-Propylamine } \\ \text { P3HT } & \text { Poly(3-hexylthiophene-2,5-diyl) } \\ \text { GO QDS } & \text { Graphene oxide quantum dots } \\ \text { NPS } & \text { Nanoparticles } \\ \text { MB } & \text { Methylene blue } \\ \text { Rb } & \text { Riboflavin } \\ \text { CMG } & \text { Chemically modified graphene } \\ \text { QDs } & \text { Quantum dots } \\ \text { FTO } & \text { Fluorene doped tin oxide } \\ \text { PPV } & \text { Poly }(p \text {-phenylenevinylene) } \\ \text { CNT } & \text { Carbon nanotube } \\ \text { SWNT } & \text { Single walled carbon nanotube } \\ \text { PANI } & \text { Polyaniline }\end{array}$

\section{Other symbols/abbreviations}

$\begin{array}{ll}\text { NIR } & \text { Near-infrared } \\ \text { HJs } & \text { Heterojunctions } \\ \text { FB } & \text { Forward bias } \\ \text { ESIPT } & \text { Intra-molecular proton transfer } \\ \text { QY } & \text { Quantum yield }\end{array}$

$\begin{array}{ll}\text { RT } & \text { Room temperature } \\ E_{\mathrm{v}}, \mathrm{VB} & \text { Valance band } \\ E_{\mathrm{c}}, \mathrm{CB} & \text { Conduction band } \\ \mathrm{VBM} & \text { Valance band maximum } \\ \lambda_{\mathrm{em}} & \text { Emission wavelength } \\ \eta & \text { Efficiency } \\ J_{\mathrm{sc}} & \text { Short-circuit current density } \\ J_{0} & \text { Saturation current } \\ V_{\mathrm{oc}} & \text { Open-circuit voltage } \\ \mathrm{FF} & \text { Fill factor } \\ \chi & \text { The electron affinity } \\ \mathrm{PCE} & \text { Power conversion efficiency } \\ \mathrm{DFT} & \text { Density functional theory } \\ V_{\mathrm{O}} & \text { Oxygen vacancy } \\ \text { Zn } & \text { Zinc interstitial } \\ \text { IOT } & \text { Indirect optical transitions } \\ \text { PR } & \text { Photoresponse } \\ \text { MO } & \text { Molecular orbital } \\ \text { PDOS } & \text { Partial density of state } \\ \text { EL } & \text { Electroluminescence } \\ \text { FET } & \text { Field-effect transistors } \\ E_{\mathrm{F}} & \text { Fermi level } \\ \mathrm{CBM} & \text { Conduction band minimum } \\ \text { LUMO } & \text { Lowest unoccupied molecular orbital } \\ \mathrm{HOMO} & \text { Highest occupied molecular orbital }\end{array}$

Instrumentation

$\begin{array}{ll}\text { CLSM } & \text { Confocal laser scanning microscopic } \\ \text { TEM } & \text { Transmission electron microscopy } \\ \text { DLS } & \text { Dynamic light scattering } \\ \text { EELS } & \text { Electron energy loss spectroscopy } \\ \text { AFM } & \text { Atomic force microscopy } \\ \text { CV } & \text { Cyclic voltammetry } \\ \text { PLE } & \text { Photoluminescence excitation } \\ \text { PDS } & \text { Photothermal deflection spectroscopy }\end{array}$

\section{Acknowledgements}

S. V. thanks The Scientific \& Technological Research Council of Turkey (TUBITAK) (TUBITAK-BIDEB 2221 - Fellowships for Visiting Scientists and Scientists on Sabbatical) for fellowship. T. U. thanks EU FP7-Marie Curie-IRG NANOWEB (PIRG06-GA2009-256428) and The Turkish Academy of Sciences - Outstanding Young Scientists Award Program (TUBA-GEBIP) for partial funding.

\section{Notes and references}

1 X. Huang, Z. Y. Yin, S. X. Wu, X. Y. Qi, Q. Y. He, Q. C. Zhang, Q. Y. Yan, F. Boey and H. Zhang, Small, 2011, 7, 1876-1902.

2 C. Chung, Y. K. Kim, D. Shin, S. R. Ryoo, B. H. Hong and D. H. Min, Acc. Chem. Res., 2013, 46, 2211-2224.

3 Y. Chen, J. Wang and Z. M. Liu, Chin. J. Anal. Chem., 2012, 40, 1772-1779. 
4 M. W. Cole, V. H. Crespi, M. S. Dresselhaus, G. Dresselhaus, J. E. Fischer, H. R. Gutierrez, K. Kojima, G. D. Mahan, A. M. Rao, J. O. Sofo, M. Tachibana, K. Wako and Q. H. Xiong, J. Phys.: Condens. Matter, 2010, 22, 334201.

5 G. Eda and M. Chhowalla, Adv. Mater., 2010, 22, 2392-2415.

6 K. P. Loh, Q. L. Bao, G. Eda and M. Chhowalla, Nat. Chem., 2010, 2, 1015-1024.

7 Y. Z. Zhou, J. Yang, X. N. Cheng, N. Zhao, H. B. Sun and D. Li, RSC Adv., 2013, 3, 3391-3398.

8 D. T. Phan, R. K. Gupta, G. S. Chung, A. A. Al-Ghamdi, O. A. Al-Hartomy, F. El-Tantawy and F. Yakuphanoglu, Sol. Energy, 2012, 86, 2961-2966.

9 B. Yin, Q. Liu, L. Yang, X. Wu, Z. Liu, Y. Hua, S. Yin and Y. Che, J. Nanosci. Nanotechnol., 2010, 10, 1934.

10 B. C. Brodie, Ann. Chim. Phys., 1860, 59, 466-472.

11 L. Staudenmaier, Ber. Dtsch. Chem. Ges., 1898, 31, 1481-1499.

12 W. Hummers and R. Offeman, J. Am. Chem. Soc., 1958, 80, 1339.

13 Z. Y. Yin, S. Y. Sun, T. Salim, S. X. Wu, X. A. Huang, Q. Y. He, Y. M. Lam and H. Zhang, ACS Nano, 2010, 4, 5263-5268.

14 B. H. Wee and J. D. Hong, Adv. Funct. Mater., 2013, 23, 4657-4666.

15 K. S. Subrahmanyam, P. Kumar, A. Nag and C. N. R. Rao, Solid State Commun., 2010, 150, 1774-1777.

16 S. Vempati, A. Shetty, P. Dawson, K. K. Nand and S. B. Krupanidhi, J. Cryst. Growth, 2012, 343, 7-12.

17 S. Vempati, J. Mitra and P. Dawson, Nanoscale Res. Lett., 2012, 7, 470 .

18 S. Vempati, S. Chirakkara, J. Mitra, P. Dawson, K. K. Nanda and S. B. Krupanidhi, Appl. Phys. Lett., 2012, 100, 162104.

19 F. Kayaci, S. Vempati, C. O. Akgun, N. Biyikli and T. Uyar, Appl. Catal., B, 2014, 156-157, 173-183.

20 F. Kayaci, S. Vempati, C. Ozgit, I. Donmez, N. Biyikli and T. Uyar, Nanoscale, 2014, 6, 5735.

21 S. Vempati, A. Shetty, P. Dawson, K. K. Nanda and S. B. Krupanidhi, Thin Solid Films, 2012, 524, 137-143.

22 F. Kayaci, S. Vempati, I. Donmez, N. Biyikli and T. Uyar, Nanoscale, 2014, 6, 10224-10234.

23 Y. Shen, S. B. Yang, P. Zhou, Q. Q. Sun, P. F. Wang, L. Wan, J. Li, L. Y. Chen, X. B. Wang, S. J. Ding and D. W. Zhang, Carbon, 2013, 62, 157-164.

24 R. Bauld, M. S. Ahmed and G. Fanchini, Phys. Status Solidi C, 2012, 9, 2374-2379.

25 X. M. Sun, Z. Liu, K. Welsher, J. T. Robinson, A. Goodwin, S. Zaric and H. J. Dai, Nano Res., 2008, 1, 203-212.

26 Z. Liu, J. T. Robinson, X. Sun and H. Dai, J. Am. Chem. Soc., 2008, 130, 10876-10877.

27 Y. W. Wang, Y. Y. Fu, Q. L. Peng, S. S. Guo, G. Liu, J. Li, H. H. Yang and G. N. Chen, J. Mater. Chem. B, 2013, 1, 5762-5767.

28 F. X. Wang, Z. Y. Gu, W. Lei, W. J. Wang, X. F. Xia and Q. L. Hao, Sens. Actuators, B, 2014, 190, 516-522.

29 X. L. Yu, G. J. Zhang, H. B. Cao, X. Q. An, Y. Wang, Z. J. Shu, X. L. An and F. Hua, New J. Chem., 2012, 36, 2593-2598.

30 E. Morales-Narvaez and A. Merkoci, Adv. Mater., 2012, 24, 3298-3308.
31 M. Nurunnabi, Z. Khatun, K. M. Huh, S. Y. Park, D. Y. Lee, K. J. Cho and Y. K. Lee, ACS Nano, 2013, 7, 6858-6867.

32 X. M. Zhou, G. Liu, J. G. Yu and W. H. Fan, J. Mater. Chem., 2012, 22, 21337-21354.

33 T. D. Nguyen-Phan, E. W. Shin, V. H. Pham, H. Kweon, S. Kim, E. J. Kim and J. S. Chung, J. Mater. Chem., 2012, 22, 20504-20511.

34 S. Essig, C. W. Marquardt, A. Vijayaraghavan, M. Ganzhorn, S. Dehm, F. Hennrich, F. Ou, A. A. Green, C. Sciascia, F. Bonaccorso, K.-P. Bohnen, H. v. Lohneysen, M. M. Kappes, P. M. Ajayan, M. C. Hersam, A. C. Ferrari and R. Krupke, Nano Lett., 2010, 10, 1589-1594.

35 T. V. Cuong, V. H. Pham, Q. T. Tran, S. H. Hahn, J. S. Chung, E. W. Shin and E. J. Kim, Mater. Lett., 2010, 64, 399-401.

36 G. Eda, Y. Y. Lin, C. Mattevi, H. Yamaguchi, H. A. Chen, I. S. Chen, C. W. Chen and M. Chhowalla, Adv. Mater., 2010, 22, 505-509.

37 D. Pan, J. Zhang, Z. Li and M. Wu, Adv. Mater., 2010, 22, 734 .

38 Z. Luo, P. M. Vora, E. J. Mele, A. T. C. Johnson and J. M. Kikkawa, Appl. Phys. Lett., 2009, 94, 111909.

39 J. L. Chen and X. P. Yan, J. Mater. Chem., 2010, 20, 4328-4332.

40 V. C. Tung, M. J. Allen, Y. Yang and R. B. Kaner, Nat. Nanotechnol., 2009, 4, 25-29.

41 J. Ito, J. Nakamura and A. Natori, J. Appl. Phys., 2008, 103, 113712.

42 K. A. Mkhoyan, A. W. Contryman, J. Silcox, D. A. Stewart, G. Eda, C. Mattevi, S. Miller and M. Chhowalla, Nano Lett., 2009, 9, 1058-1063.

43 C. Mattevi, G. Eda, S. Agnoli, S. Miller, K. A. Mkhoyan and O. Celik, Adv. Funct. Mater., 2009, 19, 2577-2583.

44 C. Gomez-Navarro, J. C. Meyer, R. S. Sundaram, A. Chuvilin, S. Kurasch, M. Burghard, K. Kern and U. Kaiser, Nano Lett., 2010, 10, 1144-1148.

45 P. Johari and V. B. Shenoy, ACS Nano, 2011, 5, 7640-7647.

46 I. Jung, D. A. Field, N. J. Clark, Y. Zhu, D. Yang, R. D. Piner, S. Stankovich, D. A. Dikin, H. Geisler, C. A. Ventrice Jr and R. S. Ruoff, J. Phys. Chem. C, 2009, 113, 18480-18486.

47 W. Gao, L. B. Alemany, L. Ci and P. M. Ajayan, Nat. Chem., 2009, 1, 403.

48 D. Li, M. B. Muller, S. Gilje, R. B. Kaner and G. G. Wallace, Nat. Nanotechnol., 2008, 3, 101.

49 V. Lee, L. Whittaker, C. Jaye, K. M. Baroudi, D. A. Fischer and S. Banerjee, Chem. Mater., 2009, 21, 3905.

50 P.-G. Ren, D.-X. Yan, X. Ji, T. Chen and Z.-M. Li, Nanotechnology, 2011, 22, 055705.

51 S. Stankovich, D. A. Dikin, R. D. Piner, K. A. Kohlhaas, A. Kleinhammes, Y. Jia, Y. Wu, S. T. Nguyen and R. S. Ruoff, Carbon, 2007, 45, 1558-1565.

52 S. P. Stankovich, R. D. Piner, X. Chen, N. Wu, S. T. Nguyen and R. S. Ruoff, J. Mater. Chem., 2006, 16, 155-158.

53 H. A. Becerril, J. Mao, Z. Liu, R. M. Stoltenberg, Z. Bao and Y. Chen, ACS Nano, 2008, 2, 464-470.

54 H. X. Chang and H. K. Wu, Adv. Funct. Mater., 2013, 23, 1984-1997. 
55 I. K. Moon, J. Lee, R. S. Ruoff and H. Lee, Nat. Commun., 2010, 1, 73.

56 A. Bagri, C. Mattevi, M. Acik, Y. J. Chabal, M. Chhowalla and V. B. Shenoy, Nat. Chem., 2010, 2, 581-587.

57 Y. A. Niu, J. P. Zhao, X. Zhang, X. J. Wang, J. Wu and Y. Li, Appl. Phys. Lett., 2012, 101, 181903.

58 C. T. Chien, S. S. Li, W. J. Lai, Y. C. Yeh, H. A. Chen, I. S. Chen, L. C. Chen, K. H. Chen, T. Nemoto, S. Isoda, M. Chen, T. Fujita, G. Eda, H. Yamaguchi, M. Chhowalla and C. W. Chen, Angew. Chem., Int. Ed., 2012, 51, 6662-6666.

59 A. Mathkar, D. Tozier, P. Cox, P. J. Ong, C. Galande, K. Balakrishnan, A. L. M. Reddy and P. M. Ajayan, J. Phys. Chem. Lett., 2012, 3, 986-991.

60 L. B. Tang, X. M. Li, R. B. Ji, K. S. Teng, G. Tai, J. Ye, C. S. Wei and S. P. Lau, J. Mater. Chem., 2012, 22, 5676-5683.

61 N. Li, M. H. Cao and C. W. Hu, Nanoscale, 2012, 4, 6205-6218.

62 S. Bai and X. P. Shen, RSC Adv., 2012, 2, 64-98.

63 Y. H. Hu, H. Wang and B. Hu, ChemSusChem, 2010, 3, 782-796.

64 X. Yan, B. Li, X. Cui, Q. Wei, K. Tajima and L. Li, J. Phys. Chem. Lett., 2011, 2, 1119-1124.

65 M. Lotya, Y. Hernandez, P. J. King, R. J. Smith, V. Nicolosi, L. S. Karlsson, F. M. Blighe, S. De, Z. Wang, I. T. McGovern, G. S. Duesberg and J. N. Coleman, J. Am. Chem. Soc., 2009, 131, 3611-3620.

66 J. T. Yuan, L. P. Ma, S. F. Pei, J. H. Du, Y. Su, W. C. Ren and H. M. Cheng, ACS Nano, 2013, 7, 4233-4241.

67 J. R. Rani, J. Lim, J. Oh, J. W. Kim, H. S. Shin, J. H. Kim, S. Lee and S. C. Jun, J. Phys. Chem. C, 2012, 116, 19010-19017.

68 T. Gokus, R. R. Nair, A. Bonetti, M. Bohmler, A. Lombardo, K. S. Novoselov, A. K. Geim, A. C. Ferrari and A. Hartschuh, ACS Nano, 2009, 3, 3963-3968.

69 Z. S. Qian, C. Wang, G. H. Du, J. Zhou, C. C. Chen, J. J. Ma, J. R. Chen and H. Feng, CrystEngComm, 2012, 14, 4976-4979.

70 M. Iliut, A. M. Gabudean, C. Leordean, T. Simon, C. M. Teodorescu and S. Astilean, Chem. Phys. Lett., 2013, 586, 127-131.

71 F. DeMichelis, S. Schreiter and A. Tagliaferro, Phys. Rev. B: Condens. Matter Mater. Phys., 1995, 51, 2143-2147.

72 R. R. Robertson and G. A. J. Amaratunga, J. Appl. Phys., 1996, 80, 2998-3003.

73 M. Koos, M. Veres, M. Fule and I. Pocsik, Diamond Relat. Mater., 2002, 11, 53-58.

74 Y. Lin, B. Zhou, R. B. Martin, K. B. Henbest, B. A. Harruff, J. E. Riggs, Z.-X. Guo, L. F. Allard and Y.-P. Sun, J. Phys. Chem. B, 2005, 109, 14779-14782.

75 Y. P. Sun, B. Zhou, Y. Lin, W. Wang, K. A. S. Fernando, P. Pathak, M. J. Meziani, B. A. Harruff, X. Wang, H. F. Wang, P. G. Luo, H. Yang, M. E. Kose, B. Chen, L. M. Veca and S. Y. Xie, J. Am. Chem. Soc., 2007, 128, 7756-7757.

76 H. Liu, T. Ye and C. Mao, Angew. Chem., Int. Ed., 2007, 46, 6473-6475.

77 S. S. Bao, Z. Hua, X. Y. Wang, Y. Zhou, C. F. Zhang, W. G. Tu, Z. G. Zou and M. Xiao, Opt. Express, 2012, 20, 28801-28807.
78 F. Yang, M. L. Zhao, B. Z. Zheng, D. Xiao, L. Wu and Y. Guo, J. Mater. Chem., 2012, 22, 25471-25479.

79 M. Melucci, M. Durso, M. Zambianchi, E. Treossi, Z. Y. Xia, I. Manet, G. Giambastiani, L. Ortolani, V. Morandi, F. De Angelis and V. Palermo, J. Mater. Chem., 2012, 22, 18237-18243.

80 W. Lv, C. H. You, S. D. Wu, B. H. Li, Z. P. Zhu, M. Z. Wang, Q. H. Yang and F. Y. Kang, Carbon, 2012, 50, 3233-3239.

81 A. Gupta and S. K. Saha, Nanoscale, 2012, 4, 6562-6567.

82 L. A. Yan, Y. N. Chang, W. Y. Yin, X. D. Liu, D. B. Xiao, G. M. Xing, L. N. Zhao, Z. J. Gu and Y. L. Zhao, Phys. Chem. Chem. Phys., 2014, 16, 1576-1582.

83 R. Thekkayil, H. John and P. Gopinath, Synth. Met., 2013, 185, 38-44.

84 M. Wojtoniszak, D. Roginska, B. Machalinski, M. Drozdzik and E. Mijowska, Mater. Res. Bull., 2013, 48, 2636-2639.

85 R. Yamuna, S. Ramakrishnan, K. Dhara, R. Devi, N. K. Kothurkar, E. Kirubha and P. K. Palanisamy, J. Nanopart. Res., 2013, 15, 1399.

86 J. Shang, L. Ma, J. Li, W. Ai, T. Yu and G. G. Gurzadyan, Sci. Rep., 2012, 2, 792.

87 X.-F. Zhang, X. Shao and S. Liu, J. Phys. Chem. A, 2012, 116, 7308.

88 C. Galande, A. D. Mohite, A. V. Naumov, W. Gao, L. Ci, A. Ajayan, H. Gao, A. Srivastava, R. B. Weisman and P. M. Ajayan, Sci. Rep., 2011, 85, 1.

89 Q. Mei, K. Zhang, G. Guan, B. Liu, S. Wang and Z. Zhang, Chem. Commun., 2010, 46, 7319-7321.

90 Z. X. Gan, S. J. Xiong, X. L. Wu, C. Y. He, J. C. Shen and P. K. Chu, Nano Lett., 2011, 11, 3951-3956.

91 V. Mamidala, L. Polavarapu, J. Balapanuru, K. P. Loh, Q. H. Xu and W. Ji, Opt. Express, 2010, 18, 25928-25935.

92 S. Wang, C. T. Nai, X. F. Jiang, Y. H. Pan, C. H. Tan, M. Nesladek, Q. H. Xu and K. P. Loh, J. Phys. Chem. Lett., 2012, 3, 2332-2336.

93 L. S. Panchakarla, K. S. Subrahmanyam, S. K. Saha, A. Govindaraj, H. R. Krishnamurthy, U. V. Waghmare and C. N. R. Rao, Adv. Mater., 2009, 21, 4726-4730.

94 S. R. P. Silva, J. Robertson Rusli, G. A. J. Amaratunga and J. Schwan, Philos. Mag. B, 1996, 74, 369-386.

95 J. Zhou, C. Booker, R. Li, X. Zhou, T.-K. Sham, X. Sun and Z. Ding, J. Am. Chem. Soc., 2007, 129, 744-745.

96 Y. Luo, X. Xia, Y. Liang, Y. Zhang, Q. Ren, J. Lia, Z. Jia and Y. Tang, J. Solid State Chem., 2007, 180, 1928-1933.

97 Y. Kanemitsu, S. Okamoto, M. Otobe and S. Oda, Phys. Rev. B: Condens. Matter Mater. Phys., 1997, 55, R7375-R7378.

98 G. Eda, C. Mattevi, H. Yamaguchi, H. Kim and M. Chhowalla, J. Phys. Chem. C, 2009, 113, 15768-15771.

99 T. V. Khai, H. G. Na, D. S. Kwak, Y. J. Kwon, H. Ham, K. B. Shim and H. W. Kim, J. Mater. Chem., 2012, 22, 17992-18003.

100 S. Standkovich, R. D. Pinter, X. Chen, N. Wu, S. B. T. Nguyen and R. S. Ruoff, J. Mater. Chem., 2006, 16, 155.

101 O. C. Compton, D. A. Dikin, K. W. Putz, L. C. Brinson and S. B. T. Nguyen, Adv. Mater., 2010, 22, 892.

102 H. K. Jeong, M. H. Jin, K. P. So, S. C. Lim and Y. H. Lee, J. Phys. D: Appl. Phys., 2009, 42, 065418. 
103 T. V. Cuong, V. H. Pham, E. W. Shin, J. S. Chung, S. H. Hur, E. J. Kim, Q. T. Tran, H. H. Nguyen and P. A. Kohl, Appl. Phys. Lett., 2011, 99, 041905.

104 S. H. Elder, F. M. Cot, Y. Su, S. M. Heald, A. M. Tyryshkin, M. K. Bowman, Y. Gao, A. G. Joly, M. L. Balmer, A. C. Kolwaite, K. A. Magrini and D. M. Blake, J. Am. Chem. Soc., 2000, 122, 5138-5146.

105 V. I. Klimov, S. A. Ivanov, J. Nanda, M. Achermann, I. Bezel, J. A. McGuire and A. Piryatinski, Nature, 2007, 447, 441-446.

106 X. F. Zhang, S. P. Liu and X. N. Shao, J. Lumin., 2013, 136, 32-37.

107 T. Yeh, F. Chan, C. Hsieh and H. Teng, J. Phys. Chem. C, 2011, 115, 22587-22597.

108 H. K. Jeong, C. Yang, B. S. Kim and K.-J. Kim, Europhys. Lett., 2010, 92, 37005.

109 R. Jose, V. Biju, Y. Yamaoka, T. Nagase, Y. Makita, Y. Shinohara, Y. Baba and M. Ishikawa, Appl. Phys. A: Mater. Sci. Process., 2004, 79, 1833-1838.

110 Y. Shen, P. Zhou, Q. Q. Sun, L. Wan, J. Li, L. Y. Chen, D. W. Zhang and X. B. Wang, Appl. Phys. Lett., 2011, 99, 141911.

111 S. N. Wang, R. Wang, X. F. Liu, X. W. Wang, D. D. Zhang, Y. J. Guo and X. H. Qiu, J. Phys. Chem. C, 2012, 116, 10702-10707.

112 J. Peng, W. Gao, B. K. Gupta, Z. Liu, R. Romero-Aburto, L. H. Ge, L. Song, L. B. Alemany, X. B. Zhan, G. H. Gao, S. A. Vithayathil, B. A. Kaipparettu, A. A. Marti, T. Hayashi, J. J. Zhu and P. M. Ajayan, Nano Lett., 2012, 12, 844-849.

113 X. M. Sun, D. C. Luo, J. F. Liu and D. G. Evans, ACS Nano, 2010, 4, 3381-3389.

114 F. Tuinstra and J. L. Koenig, J. Chem. Phys., 1970, 53, 1126.

115 D. S. Knight and W. B. White, J. Mater. Res., 1989, 4, 385-393.

116 Z. J. Zhang, S. Fan, J. L. Huang and C. M. Lieber, J. Electron. Mater., 1996, 25, 57-61.

117 H. Wehrheit, T. Au, R. Schmechel, S. O. Shalamberidze, G. I. Kalandadze and A. M. Eristavi, J. Solid State Chem., 1999, 154, 79-86.

118 K. Shirai, S. Emura, S. Gonda and Y. Kumashiro, J. Appl. Phys., 1995, 78, 3392-3398.

119 S. J. Henley, J. D. Carey and S. R. P. Silva, Appl. Phys. Lett., 2004, 85, 6236.

120 F. C. Tai, C. Wei, S. H. Chang and W. S. Chen, J. Raman Spectrosc., 2010, 41, 933-937.

121 K. Erickson, R. Erni, Z. Lee, N. Alem, W. Gannett and A. Zettl, Adv. Mater., 2010, 22, 4467-4472.

122 D. V. C. Melnikov and J. R. Chelikowsky, Phys. Rev. Lett., 2004, 92, 046802.

123 D. Bourissou, O. Guerret, F. P. Gabbai and G. Bertrand, Chem. Rev., 2000, 100, 39-91.

124 R. Hoffmann, J. Am. Chem. Soc., 1968, 90, 1475-1476.

125 J. R. Rani, J. Lim, J. Oh, D. Kim, D. Lee, J. W. Kim, H. S. Shin, J. H. Kim and S. C. Jun, RSC Adv., 2013, 3, 5926-5936.

126 R. Ishikawa, M. Bando, Y. Morimoto and A. Sandhu, Nanoscale Res. Lett., 2011, 6, 111.

127 R. Ishikawa, P. J. Ko, M. Bando, Y. Kurokawa, A. Sandhu and M. Konagai, Nanoscale Res. Lett., 2013, 8, 534.
128 T. V. Khai, H. G. Na, D. S. Kwak, Y. J. Kwon, H. Ham, K. B. Shim and H. W. Kim, Chem. Eng. J., 2012, 211, 369-377.

129 L. B. Tang, R. B. Ji, X. M. Li, K. S. Teng and S. P. Lau, J. Mater. Chem. C, 2013, 1, 4908-4915.

130 F. Karlicky, K. K. R. Datta, M. Otyepka and R. Zboril, ACS Nano, 2013, 7, 6434-6464.

131 T. W. Lin, C. Y. Su, X. Q. Zhang, W. J. Zhang, Y. H. Lee, C. W. Chu, H. Y. Lin, M. T. Chang, F. R. Chen and L. J. Li, Small, 2012, 8, 1384-1391.

132 R. Schmechel, H. Werheit, T. U. Kampen and W. Mönch, J. Solid State Chem., 2004, 177, 566-568.

133 H. Werheit, H. W. Rotter, S. Shalamberidze, A. L. Jasper and T. Tanaka, Phys. Status Solidi B, 2011, 248, 1275-1279.

134 Y. Z. Lu, Y. Y. Jiang, W. T. Wei, H. B. Wu, M. M. Liu, L. Niu and W. Chen, J. Mater. Chem., 2012, 22, 2929-2934.

135 Z.-B. Liu, Y.-F. Xu, X.-Y. Zhang, X.-L. Zhang, Y.-S. Chen and J.-G. Tian, J. Phys. Chem. B, 2009, 113, 9681-9686.

136 Y. F. Xu, Z. B. Liu, X. L. Zhang, Y. Wang, J. G. Tian, Y. Huang, Y. F. Ma, X. Y. Zhang and Y. S. Chen, Adv. Mater., 2009, 21, 1275.

137 Y. S. Liu, J. Y. Zhou, X. L. Zhang, Z. B. Liu, X. J. Wan, J. G. Tian, T. Wang and Y. S. Chen, Carbon, 2009, 47, 3113-3121.

138 J. H. Jung, D. S. Cheon, F. Liu, K. B. Lee and T. S. Seo, Angew. Chem., Int. Ed., 2010, 49, 5708.

139 S. K. Singh, M. K. Singh, M. K. Nayak, S. Kumari, J. J. A. Gracio and D. Dash, Carbon, 2011, 49, 684-692.

140 F. Liu, J. Y. Choi and T. S. Seo, Biosens. Bioelectron., 2010, 25, 2361.

141 S. H. Alarcon, D. Pagani, J. Bacigalupo and A. C. Olivieri, J. Mol. Struct., 1999, 475, 233.

142 Z. B. Liu, Y. Wang, X. L. Zhang, Y. F. Xu, Y. S. Chen and J. G. Tian, Appl. Phys. Lett., 2009, 94, 021902.

143 J. J. Wang, M. Feng and H. B. Zhan, Opt. Laser Technol., 2014, 57, 84-89.

144 X. Q. Zheng, M. Feng and H. B. Zhan, J. Mater. Chem. C, 2013, 1, 6759-6766.

145 X. L. Zhang, Z. B. Liu, X. C. Li, Q. Ma, X. D. Chen, J. G. Tian, Y. F. Xu and Y. S. Chen, Opt. Express, 2013, 21, 7511-7520.

146 S. Kumar, M. Anija, N. Kamaraju, K. S. Vasu, K. S. Subrahmanyam, A. K. Sood and C. N. R. Rao, Appl. Phys. Lett., 2009, 95, 191911.

147 P. Chantharasupawong, R. Philip, N. T. Narayanan, P. M. Sudeep, A. Mathkar, P. M. Ajayan and J. Thomas, J. Phys. Chem. C, 2012, 116, 25955-25961.

148 B. L. Allen, P. D. Kichambare, P. Gou, I. I. Vlasova, A. A. Kapralov, N. Konduru, V. E. Kagan and A. Star, Nano Lett., 2008, 8, 3899.

149 J. L. Chen and X. P. Yan, Chem. Commun., 2011, 47, 3135. 150 L. Minati, G. Speranza, I. Bernagozzi, S. Torrengo, L. Toniutti, B. Rossi, M. Ferrari and A. Chiasera, J. Phys. Chem. C, 2010, 114, 11068.

151 J. Lu, J. Yang, J. Wang, A. Lim, S. Wang and K. P. Loh, ACS Nano, 2009, 3, 2367-2375.

152 S. Kochmann, T. Hirsch and O. S. Wolfbeis, J. Fluoresc., 2012, 22, 849 . 
153 W. Zhao, C. Song and P. E. Pehrsson, J. Am. Chem. Soc., 2002, 124, 12418.

154 Y. Chen and Z. Rosenzweig, Anal. Chem., 2002, 74, 5132-5138.

155 L.-J. Fan, Y. Zhang, C. B. Murphy, S. E. Angell, M. F. L. Parker, B. R. Flynn and W. E. Jones Jr, Coord. Chem. Rev., 2009, 253, 410-422.

156 C. H. Fan, S. Wang, J. W. Hong, G. C. Bazan, K. W. Plaxco and A. J. Heeger, Proc. Natl. Acad. Sci. U. S. A., 2003, 100, 6297-6301.

157 W. W. Tu, J. P. Lei, S. Y. Zhang and H. X. Ju, Chem. - Eur. J., 2010, 16, 10771-10777.

158 B. K. Gupta, P. Thanikaivelan, T. N. Narayanan, L. Song, W. Gao, T. Hayashi, A. L. M. Reddy, A. Saha, V. Shanker, M. Endo, A. A. Marti and P. M. Ajayan, Nano Lett., 2011, 11, 5227-5233.

159 G. G. Blasse and B. C. Grabmaier, Luminescent Materials, Springer, New York, 1994.

160 L. F. Gomes, K. T. de Oliveira, C. R. Neri, P. C. de Sousa Filho, M. J. Dal Bianco, A. P. Ramos, M. E. D. Zaniquelli and O. A. Serra, J. Lumin., 2008, 128, 1339-1347.

161 R. D. Ionescu and T. Frejd, J. Lumin., 2004, 106, 133-139.

162 Z. L. Mo, Y. X. Zhao, R. B. Guo, P. W. Liu and T. T. Xie, Mater. Manuf. Processes, 2012, 27, 494-498.

163 Z. L. Yang, X. J. Shi, J. J. Yuan, H. T. Pu and Y. S. Liu, Appl. Surf. Sci., 2010, 257, 138-142.

164 J. Zou, L. Liu, H. Chen, S. I. Khondaker, R. D. McCullough, Q. Huo and L. Zhai, Adv. Mater., 2008, 20, 2055-2060.

165 Y. Liang, D. Wu, X. Feng and K. Mullen, Adv. Mater., 2009, 21, 1-5.

166 Q. Su, S. Pang, V. Alijani, C. Li, X. Feng and K. Mullen, Adv. Mater., 2009, 21, 3191-3195.

167 S. D. Stranks, C. Weisspfennig, P. Parkinson, M. B. Johnston, L. M. Herz and R. J. Nicholas, Nano Lett., 2011, 11, 66.

168 Y. Tachibana, J. E. Moser, M. Grätzel, D. R. Klug and J. R. Durrant, J. Phys. Chem., 1996, 100, 20056.

169 D. L. Meng, J. H. Sun, S. D. Jiang, Y. Zeng, Y. Li, S. K. Yan, J. X. Geng and Y. Huang, J. Mater. Chem., 2012, 22, 21583-21591.

170 S. Goswami, U. N. Maiti, S. Maiti, S. Nandy, M. K. Mitra and K. K. Chattopadhyay, Carbon, 2011, 49, 2245-2252.

171 Z. G. Tang, Q. M. Liu, I. Khatri, R. Ishikawa, K. Ueno and H. Shirai, in Phys. Status Solidi (c), ed. S. Pizzini, G. Kissinger, H. YamadaKaneta and J. Kang, Wiley-VCH Verlag Gmbh, Weinheim, 2012, vol. 9, pp. 2075-2078.

172 J. J. Fan, S. W. Liu and J. G. Yu, J. Mater. Chem., 2012, 22, 17027-17036.

173 J. M. Kroon, N. J. Bakker, H. J. P. Smit, P. Liska, K. R. Thampi, P. Wang, S. M. Zakeeruddin, M. Gratzel, A. Hinsch, S. Hore, U. Wurfel, R. Sastrawan, J. R. Durrant, E. Palomares, H. Pettersson, T. Gruszecki, J. Walter, K. Skupien and G. E. Tulloch, Prog. Photovolt.: Res. Appl., 2007, 15, 1-18.

174 H. F. Liang, W. Ren, J. H. Su and C. L. Cai, Thin Solid Films, 2012, 521, 163-167.

175 G. D. Sharma, M. L. Keshtov, A. R. Khokhlov, D. Tasis and C. Galiotis, Org. Electron., 2014, 15, 348-355.
176 H. X. Chang, Z. H. Sun, M. Saito, Q. H. Yuan, H. Zhang, J. H. Li, Z. C. Wang, T. Fujita, F. Ding, Z. J. Zheng, F. Yan, H. K. Wu, M. W. Chen and Y. Ikuhara, ACS Nano, 2013, 7, 6310-6320.

177 Y. Lin, K. Zhang, W. F. Chen, Y. D. Liu, Z. G. Geng, J. Zeng, N. Pan, L. F. Yan, X. P. Wang and J. G. Hou, ACS Nano, 2010, 4, 3033-3038.

178 S. M. Z. Islam, T. Gayen, M. Seredych, T. J. Bandosz and R. Alfano, Optical Components and Materials X, 2013, 8621, 86211T.

179 F. Zou, Y. A. Yu, N. Cao, L. Z. Wu and J. F. Zhi, Scr. Mater., 2011, 64, 621-624.

180 H. Q. Sun, S. Z. Liu, S. M. Liu and S. B. Wang, Appl. Catal., $B, 2014,146,162-168$.

181 N. Farhangi, R. R. Chowdhury, Y. Medina-Gonzalez, M. B. Ray and P. A. Charpentier, Appl. Catal., B, 2011, 110, 25-32.

182 V. Stengl, J. Henych, P. Vomacka and M. Slusna, Photochem. Photobiol., 2013, 89, 1038-1046.

183 G. Singh, A. Choudhary, D. Haranath, A. G. Joshi, N. Singh, S. Singh and R. Pasricha, Carbon, 2011, 50, 385-394.

184 S. Ameen, M. S. Akhtar, H. K. Seo and H. S. Shin, Mater. Lett., 2013, 100, 261-265.

185 H. R. Pant, B. Pant, H. J. Kim, A. Amarjargal, C. H. Park, L. D. Tijing, E. K. Kim and C. S. Kim, Ceram. Int., 2013, 39, 5083-5091.

186 M. Ahmad, E. Ahmed, Z. L. Hong, N. R. Khalid, W. Ahmed and A. Elhissi, J. Alloys Compd., 2013, 577, 717-727.

187 M. Y. Wang, J. R. Huang, Z. W. Tong, W. H. Li and J. Chen, J. Alloys Compd., 2013, 568, 26-35.

188 P. Wang, T. Jiang, C. Zhu, Y. Zhai, D. Wang and S. Dong, Nano Res., 2010, 3, 794.

189 S. Kaveri, L. Thirugnanam, M. Dutta, J. Ramasamy and N. Fukata, Ceram. Int., 2013, 39, 9207-9214.

190 R. C. Pawar and C. S. Lee, Mater. Chem. Phys., 2013, 141, 686-693.

191 H. T. Hu, X. B. Wang, F. M. Liu, J. C. Wang and C. H. Xu, Synth. Met., 2011, 161, 404-410.

192 A. F. Zedan, S. Sappal, S. Moussa and M. S. El-Shall, J. Phys. Chem. C, 2010, 114, 19920-19927.

193 J. Chu, X. Li and P. Xu, J. Mater. Chem., 2011, 21, 11283-11287.

194 G. Y. Chen, D. J. Wang, C. Liang, Z. Y. Wei, W. X. Zhang, J. C. Liang and D. M. Wang, Rare Met. Mater. Eng., 2012, 41, 1153-1155.

195 H. G. Wei, J. H. Zhu, S. J. Wu, S. Y. Wei and Z. H. Guo, Polymer, 2013, 54, 1820-1831.

196 D. I. Son, B. W. Kwon, D. H. Park, W. S. Seo, Y. Yi, B. Angadi, C. L. Lee and W. K. Choi, Nat. Nanotechnol., 2012, 7, 465-471.

197 H. D. Zeng, Y. Cao, S. F. Xie, J. H. Yang, Z. H. Tang, X. Y. Wang and L. Y. Sun, Nanoscale Res. Lett., 2013, 8, 133.

198 S. Vempati, Y. Ertas and T. Uyar, J. Phys. Chem. C, 2013, 17, 21609-21618.

199 J. J. Ding, M. Q. Wang, J. P. Deng, W. Y. Gao, Z. Yang, C. X. Ran and X. Y. Zhang, J. Alloys Compd., 2014, 582, 29-32.

200 G. Williams and P. V. Kamat, Langmuir, 2009, 25, 13869-13873. 
201 B. Wei, M. Hsu, P. Su, H. Lin, R. Wu and H. A. Lai, Sens. Actuators, B, 2004, 101, 81-89.

202 L. L. Zhang, R. Zhou and X. S. Zhao, J. Mater. Chem., 2010, 20, 5983-5992.

203 N. Zhang, Y. H. Zhang, X. Y. Pan, X. Z. Fu, S. Q. Liu and Y. J. Xu, J. Phys. Chem. C, 2011, 115, 23501-23511.

204 C. Nethravathi, T. Nisha, N. Ravishankar, C. Shivakumara and M. Rajamathi, Carbon, 2009, 47, 2054-2059.

205 B. T. Raut, M. A. Chougule, S. Sen, R. C. Pawar, C. S. Lee and V. B. Patil, Ceram. Int., 2012, 38, 3999-4007.

206 A. Cao, Z. Liu, S. Chu, M. Wu, Z. Ye, Z. Cai, Y. Chang, S. Wang, Q. Gong and Y. Liu, Adv. Mater., 2010, 22, 103.

207 Z. Lu, C. X. Guo, H. B. Yang, Y. Qiao, J. Guo and C. M. Li, J. Colloid Interface Sci., 2011, 353, 588.

208 H. Dong, W. Gao, F. Yan, H. Ji and H. Ju, Anal. Chem., 2010, 82, 5511.
209 T. Sasaki and M. Watanabe, J. Phys. Chem. B, 1997, 101, 10159-10161.

210 N. D. Abazovic, M. I. Comor, M. D. Dramicanin, D. J. Jovanovic, S. P. Ahrenkiel and J. M. Nedeljkovic, J. Phys. Chem. B, 2006, 110, 25366-25370.

211 Y. T. Liang, B. K. Vijayan, K. A. Gray and M. C. Hersam, Nano Lett., 2011, 11, 2865-2870.

212 W. Tu, Y. Zhou, Q. Liu, Z. Tian, J. Gao, X. Chen, H. Zhang, J. Liu and Z. Zou, Adv. Funct. Mater., 2012, 22, 1215-1221.

213 L. Y. Cheng, J. Zhou, Q. Zou, Y. T. Long and H. Tian, RSC Adv., 2012, 2, 4623-4626.

214 N. Karousis, A. S. D. Sandanayaka, T. Hasobe, S. P. Economopoulos, E. Sarantopoulou and N. Tagmatarchis, J. Mater. Chem., 2011, 21, 109.

215 R. Stalder, C. Grand, J. Subbiah, F. So and J. R. Reynolds, Polym. Chem., 2012, 3, 89. 